\title{
Duality-invariant superconformal higher-spin models
}

\author{
Sergei M. Kuzenko $\odot^{*}$ and Emmanouil S. N. Raptakis $\circledast^{\dagger}$ \\ Department of Physics M013, The University of Western Australia, \\ 35 Stirling Highway, Perth, Western Australia 6009, Australia
}

(Received 28 July 2021; accepted 6 October 2021; published 3 December 2021)

\begin{abstract}
We develop a general formalism of duality rotations for bosonic conformal spin- $s$ gauge fields, with $s \geq 2$, in a conformally flat four-dimensional spacetime. In the $s=1$ case, this formalism is equivalent to the theory of $\mathrm{U}(1)$ duality-invariant nonlinear electrodynamics developed by Gaillard and Zumino, Gibbons and Rasheed, and generalized by Ivanov and Zupnik. For each integer spin $s \geq 2$ we demonstrate the existence of families of conformal U(1) duality-invariant models, including a generalization of the so-called ModMax electrodynamics $(s=1)$. Our bosonic results are then extended to the $\mathcal{N}=1$ and $\mathcal{N}=2$ supersymmetric cases. We also sketch a formalism of duality rotations for conformal gauge fields of Lorentz type $(m / 2, n / 2)$, for positive integers $m$ and $n$.
\end{abstract}

DOI: 10.1103/PhysRevD.104.125003

\section{INTRODUCTION}

Building on the seminal 1981 work by Gaillard and Zumino [1], the general theory of U(1) duality-invariant models for nonlinear electrodynamics in four dimensions was developed in the mid 1990s [2-5] and the early 2000s [6-8]. The formalism of [2-5] has been generalized to formulate general $\mathrm{U}(1)$ duality-invariant $\mathcal{N}=1$ and $\mathcal{N}=2$ globally $[9,10]$ and locally [11-13] supersymmetric theories. In particular, Ref. [9] put forward the constructive perturbative scheme to compute $\mathcal{N}=2$ superconformal $\mathrm{U}(1)$ duality-invariant actions for the $\mathcal{N}=2$ vector multiplet. Moreover, extending the earlier proposal of [14], the first consistent perturbative scheme to construct the $\mathcal{N}=2$ supersymmetric Born-Infeld action was given in [10]. The formalism of nonlinear realizations for the partial $\mathcal{N}=4 \rightarrow \mathcal{N}=2$ breaking of supersymmetry advocated in [15] reproduced [16] the results of [10]. Further progress toward the construction of the $\mathcal{N}=2$ supersymmetric Born-Infeld action has been achieved in [17-19]. ${ }^{1}$

\footnotetext{
*sergei.kuzenko@uwa.edu.au

emmanouil.raptakis@research.uwa.edu.au

${ }^{1}$ It should be pointed out that the $\mathcal{N}=1$ supersymmetric Born-Infeld action [20] is the first nontrivial U(1) dualityinvariant supersymmetric theory [21]. Its remarkable property is that it is a Goldstone multiplet action for partial $\mathcal{N}=2 \rightarrow$ $\mathcal{N}=1$ supersymmetry breaking in Minkowski space [22,23], as well as in the maximally supersymmetric backgrounds [24] discovered in [25]: (i) $\mathbb{R} \times S^{3}$; (ii) $\mathrm{AdS}_{3} \times \mathbb{R}$; and (iii) a supersymmetric plane wave.

Published by the American Physical Society under the terms of the Creative Commons Attribution 4.0 International license. Further distribution of this work must maintain attribution to the author(s) and the published article's title, journal citation, and DOI. Funded by SCOAP ${ }^{3}$.
}

Within the original bosonic formulation [2-5] and its supersymmetric extensions [9,10], U(1) duality invariance of a model for nonlinear (super) electrodynamics is equivalent to the condition that the Lagrangian satisfies a nonlinear self-duality equation. General solutions of such equations are difficult to find. Ivanov and Zupnik [6-8] provided a reformulation of nonlinear electrodynamics which makes use of certain auxiliary variables in such a way that (i) the self-interaction depends only on the auxiliary variables; and (ii) $\mathrm{U}(1)$ duality invariance is equivalent to the manifest $\mathrm{U}(1)$ invariance of the self-interaction. Supersymmetric extensions of the Ivanov-Zupnik approach were given in $[26,27]$. In particular, the $\mathcal{N}=2$ supersymmetric formulation of [26] has been used to obtain the closed-form expression for a superconformal $\mathrm{U}(1)$ duality-invariant model proposed to describe the low-energy effective action for $\mathcal{N}=4$ superYang-Mills theory [28].

In this paper we will demonstrate that the known results for U(1) duality-invariant nonlinear electrodynamics ( $\operatorname{spin} s=1$ ) can naturally be generalized to develop a general formalism of $\mathrm{U}(1)$ duality rotations for bosonic conformal spin- $s$ gauge fields, with $s \geq 2$, and their $\mathcal{N}=1$ and $\mathcal{N}=2$ supersymmetric cousins in a conformally flat four-dimensional background.

Our paper is organized as follows. In Sec. II we introduce the notion of $\mathrm{U}(1)$ duality-invariant conformal higherspin (CHS) theories and present examples of such models, including higher-spin generalizations of the so-called "ModMax electrodynamics" [29] (see also [30]). This purely bosonic study is extended in Sec. III to the case of $\mathcal{N}=1$ superconformal higher-spin (SCHS) multiplets. We present a one-parameter self-dual SCHS action, which generalizes the $\mathcal{N}=1$ superconformal $U(1)$ dualityinvariant electrodynamics $[28,31]$. In Sec. IV we uplift the 
technical machinery of the previous two sections to $\mathcal{N}=2$ superspace and derive the self-duality equation for $\mathcal{N}=2$ SCHS models. In Sec. $\mathrm{V}$ we provide concluding comments and sketch the formalism of duality rotations for CHS gauge fields of arbitrary rank. The main body of this paper is accompanied by four technical appendixes. Appendix A reviews the salient details of conformal geometry in four dimensions pertinent to this work. Appendixes $\mathrm{B}$ and $\mathrm{C}$ review the relevant aspects of $\mathcal{N}=1$ and $\mathcal{N}=2$ conformal superspace, respectively. In Appendix D we derive a class of duality-invariant CHS models via the auxiliary field approach. In Appendix E we provide arguments to fix the overall signs of the free (super) conformal higher-spin actions.

\section{DUALITY-INVARIANT CHS MODELS}

In this section we develop a formalism of duality rotations for CHS fields and propose some duality-invariant models.

Consider a dynamical system describing the propagation of a conformal spin- $s$ field $h_{\alpha(s) \dot{\alpha}(s)}:=h_{\alpha_{1} \ldots \alpha_{s} \dot{\alpha}_{1} \ldots \dot{\alpha}_{s}}=$ $h_{\left(\alpha_{1} \ldots \alpha_{s}\right)\left(\dot{\alpha}_{1} \ldots \dot{\alpha}_{s}\right)}$, with $s \geq 1$, in curved spacetime. Its action functional $S^{(s)}[\mathcal{C}, \overline{\mathcal{C}}]$ is assumed to depend on a field strength $\mathcal{C}_{\alpha(2 s)}$ and its conjugate $\overline{\mathcal{C}}_{\dot{\alpha}(2 s)}$, with $\mathcal{C}_{\alpha(2 s)}$ being defined as

$$
\mathcal{C}_{\alpha(2 s)}=\nabla_{\left(\alpha_{1}\right.} \dot{\beta}_{1} \ldots \nabla_{\alpha_{s}} \dot{\beta}_{s} h_{\left.\alpha_{s+1} \ldots \alpha_{2 s}\right) \dot{\beta}(s)},
$$

where $\nabla_{a}$ denotes the conformally covariant derivative, see Appendix A. The real unconstrained prepotential $h_{\alpha(s) \dot{\alpha}(s)}$ is a primary field, $K_{b} h_{\alpha(s) \dot{\alpha}(s)}=0$, where $K_{b}$ is the special conformal generator. It is defined modulo gauge transformations of the form

$$
\delta_{\zeta} h_{\alpha(s) \dot{\alpha}(s)}=\nabla_{\left(\alpha _ { 1 } \left(\dot{\alpha}_{1}\right.\right.} \zeta_{\left.\left.\alpha_{2} \ldots \alpha_{s}\right) \dot{\alpha}_{2} \ldots \dot{\alpha}_{s}\right)},
$$

with the gauge parameter $\zeta_{\alpha(s-1) \dot{\alpha}(s-1)}$ also being primary. This transformation law is conformally invariant provided

$$
\mathbb{D} h_{\alpha(s) \dot{\alpha}(s)}=(2-s) h_{\alpha(s) \dot{\alpha}(s)},
$$

where $\mathbb{D}$ is the dilatation generator. The field strength (2.1) is primary in a generic gravitational background,

$$
K_{b} \mathcal{C}_{\alpha(2 s)}=0, \quad \mathbb{D} \mathcal{C}_{\alpha(2 s)}=2 \mathcal{C}_{\alpha(2 s)} .
$$

However, the gauge transformations (2.2) leave $\mathcal{C}_{\alpha(2 s)}$ invariant only when $s=1, \delta_{\zeta} \mathcal{C}_{\alpha(2)}=0$. For $s \geq 2$ gauge invariance holds only if the background is conformally flat,

$$
C_{\alpha(4)}=0 \Rightarrow \delta_{\zeta} \mathcal{C}_{\alpha(2 s)}=0,
$$

where $C_{\alpha(4)}$ is the self-dual part of the background Weyl tensor, see Appendix A. For the remainder of this section we will assume such a geometry.

We point out that $\mathcal{C}_{\alpha(2)}$ is Maxwell's field strength and $\mathcal{C}_{\alpha(4)}$ is the linearized Weyl tensor. We will refer to $\mathcal{C}_{\alpha(2 s)}$ for $s>2$ as the linearized spin-s Weyl tensor.

\section{A. U(1) duality-invariant models}

It is important to note that the field strength (2.1) obeys the Bianchi identity

$$
\nabla^{\beta_{1}} \dot{\alpha}_{1} \ldots \nabla^{\beta_{s}} \dot{\alpha}_{s} \mathcal{C}_{\alpha(s) \beta(s)}=\nabla_{\left(\alpha_{1}\right.} \dot{\beta}_{1} \ldots \nabla_{\left.\alpha_{s}\right)} \dot{\beta}_{s} \overline{\mathcal{C}}_{\dot{\alpha}(s) \dot{\beta}(s)} .
$$

Now, we assume that $S^{(s)}[\mathcal{C}, \overline{\mathcal{C}}]$ is extended to be a functional of an unconstrained field $\mathcal{C}_{\alpha(2 s)}$ and its conjugate. We introduce

$$
\mathrm{i} \mathcal{M}_{\alpha(2 s)}:=\frac{\delta S^{(s)}[\mathcal{C}, \overline{\mathcal{C}}]}{\delta \mathcal{C}^{\alpha(2 s)}}
$$

where we have defined

$$
\delta S^{(s)}[\mathcal{C}, \overline{\mathcal{C}}]=\int \mathrm{d}^{4} x e \delta \mathcal{C}^{\alpha(2 s)} \frac{\delta S^{(s)}[\mathcal{C}, \overline{\mathcal{C}}]}{\delta \mathcal{C}^{\alpha(2 s)}}+\text { c.c. }
$$

Varying $S^{(s)}[\mathcal{C}, \overline{\mathcal{C}}]$ with respect to the prepotential $h_{\alpha(s) \dot{\alpha}(s)}$ yields

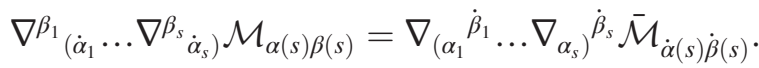

A crucial feature of our analysis above is that the functional form of the equation of motion (2.9) mirrors that of the Bianchi identity (2.6). Consequently, the union of Eqs. (2.6) and (2.9) is invariant under the $\mathrm{SO}(2) \cong \mathrm{U}(1)$ duality transformations:

$$
\delta_{\lambda} \mathcal{C}_{\alpha(2 s)}=\lambda \mathcal{M}_{\alpha(2 s)}, \quad \delta_{\lambda} \mathcal{M}_{\alpha(2 s)}=-\lambda \mathcal{C}_{\alpha(2 s)},
$$

where $\lambda$ is a constant, real parameter. One may then obtain two equivalent expressions for the variation of $S^{(s)}[\mathcal{C}, \overline{\mathcal{C}}]$ with respect to (2.10)

$$
\begin{aligned}
\delta_{\lambda} S^{(s)}[\mathcal{C}, \overline{\mathcal{C}}] & =\frac{\mathrm{i} \lambda}{4} \int \mathrm{d}^{4} x e\left\{\mathcal{C}^{2}-\mathcal{M}^{2}\right\}+\text { c.c. } \\
& =-\frac{\mathrm{i} \lambda}{2} \int \mathrm{d}^{4} x e \mathcal{M}^{2}+\text { c.c. }
\end{aligned}
$$

as a generalization of similar derivations in nonlinear electrodynamics $[4,5,10]$. This implies the self-duality equation 


$$
\operatorname{Im} \int \mathrm{d}^{4} x e\left\{\mathcal{C}^{\alpha(2 s)} \mathcal{C}_{\alpha(2 s)}+\mathcal{M}^{\alpha(2 s)} \mathcal{M}_{\alpha(2 s)}\right\}=0
$$

which must hold for an unconstrained field $\mathcal{C}_{\alpha(2 s)}$ and its conjugate. In (2.11) we have employed the notational shorthand $T^{2}=T^{\alpha(m)} T_{\alpha(m)}$ (similarly $\bar{T}^{2}=\bar{T}_{\dot{\alpha}(m)} \bar{T}^{\dot{\alpha}(m)}$ ). The simplest solution of the self-duality equation (2.12) is the free CHS model (E1), which was introduced in [32-34] in the case of Minkowski space and extended to arbitrary conformally flat backgrounds in [35]. ${ }^{2}$

In the $s=1$ case, the self-duality equation (2.12) was originally derived by Bialynicki-Birula [36], but unfortunately this work was largely unnoticed. ${ }^{3}$ It was independently re-discovered by Gibbons and Rasheed in 1995 [2]. Two years later, it was rederived by Gaillard and Zumino [4] with the aid of their formalism developed back in 1981 [1] but originally applied only in the linear case.

As is known, all gravity-matter theories allow for a Weylinvariant formulation $[37,38]$ in which the gravitational field is described in terms of two gauge fields. One of them is the inverse vielbein $e_{a}{ }^{m}$ and the other is a conformal compensator $\Psi$, the latter being a nowhere vanishing scalar field. In this setting the gravity gauge group also includes Weyl transformations, which act on the gravitational fields as follows:

$$
e_{a}{ }^{m} \rightarrow \mathrm{e}^{\sigma} e_{a}{ }^{m}, \quad \Psi \rightarrow \mathrm{e}^{\sigma} \Psi .
$$

Truly conformal theories, such as conformal gravity, do not depend on the compensator. In the approach of [39], the gauge group is further enlarged to local conformal transformations, and the compensator is a primary dimension-1 scalar field,

$$
K_{b} \Psi=0, \quad \mathbb{D} \Psi=\Psi .
$$

If we allow for the action $S^{(s)}[\mathcal{C}, \overline{\mathcal{C}}]$ to depend on the compensator, then the family of $\mathrm{U}(1)$ duality-invariant theories (2.12) is very large. For instance, the following $\mathrm{U}(1)$ duality-invariant model

$$
\begin{aligned}
S_{\mathrm{BI}}^{(s)}[\mathcal{C}, \overline{\mathcal{C}} ; \Psi]= & -\int \mathrm{d}^{4} x e \Psi^{4}\left\{1-\left(1+(-1)^{s} \frac{\mathcal{C}^{2}+\overline{\mathcal{C}}^{2}}{\Psi^{4}}\right.\right. \\
& \left.\left.+\frac{\left(\mathcal{C}^{2}-\mathcal{C}^{2}\right)^{2}}{4 \Psi^{8}}\right)^{\frac{1}{2}}\right\}
\end{aligned}
$$

is a higher-spin generalization of Born-Infeld electrodynamics [40]. The latter is obtained from (2.15) for $s=1$ by making use of local scale transformations to impose a gauge condition $\Psi^{2}=g^{-1}=$ const. Owing to the dependence of $S_{\mathrm{BI}}^{(s)}[\mathcal{C}, \overline{\mathcal{C}} ; \Psi]$ on the compensator $\Psi$, it is clear that (2.15) is not conformal.

As another solution of the self-duality equation (2.12), we propose a one-parameter duality-invariant extension of (2.15)

$$
S_{\text {BIgen }}^{(s)}[\mathcal{C}, \overline{\mathcal{C}} ; \Psi]=-\int \mathrm{d}^{4} x e \Psi^{4}\left\{1-\left(1+\frac{2}{\Psi^{4}}\left[\frac{(-1)^{s}}{2} \cosh \gamma\left(\mathcal{C}^{2}+\overline{\mathcal{C}}^{2}\right)+\sinh \gamma\left(\mathcal{C}^{2} \overline{\mathcal{C}}^{2}\right)^{\frac{1}{2}}\right]+\frac{\left(\mathcal{C}^{2}-\mathcal{C}^{2}\right)^{2}}{4 \Psi^{8}}\right)^{\frac{1}{2}}\right\}, \gamma \in \mathbb{R}
$$

For $s=1$ this model was introduced in [41].

\section{B. Self-duality under Legendre transformation}

In the case of nonlinear (super) electrodynamics, U(1) duality invariance implies self-duality under Legendre transformations, see [10] for a review. This remarkable property proves to extend to the higher-spin case, as will be shown below.

We start by describing a Legendre transformation for a generic theory with action $S^{(s)}[\mathcal{C}, \overline{\mathcal{C}}]$. For this we introduce the parent action

$$
\begin{aligned}
S^{(s)} & {\left[\mathcal{C}, \overline{\mathcal{C}}, \mathcal{C}^{\mathrm{D}}, \overline{\mathcal{C}}^{\mathrm{D}}\right] } \\
& =S^{(s)}[\mathcal{C}, \overline{\mathcal{C}}]+\int \mathrm{d}^{4} x e\left(\frac{\mathrm{i}}{2} \mathcal{C}^{\alpha(2 s)} \mathcal{C}_{\alpha(2 s)}^{\mathrm{D}}+\text { c.c. }\right) .
\end{aligned}
$$

\footnotetext{
${ }^{2}$ For the free CHS model (E1), one can also consider scale transformations in addition to the U(1) duality ones (2.10), which is similar to the case of electrodynamics discussed, e.g., in [10].

${ }^{3}$ We thank Dmitri Sorokin for bringing Ref. [36] to our attention.
}

Here $\mathcal{C}_{\alpha(2 s)}$ is an unconstrained field and $\mathcal{C}_{\alpha(2 s)}^{\mathrm{D}}$ takes the form

$$
\mathcal{C}_{\alpha(2 s)}^{\mathrm{D}}=\nabla_{\left(\alpha_{1}\right.}^{\dot{\beta}_{1}} \ldots \nabla_{\alpha_{s}}^{\dot{\beta}_{s}} h_{\left.\alpha_{s+1} \ldots \alpha_{2 s}\right) \dot{\beta}(s)}^{\mathrm{D}},
$$

where $h_{\alpha(s) \dot{\alpha}(s)}^{\mathrm{D}}$ is a Lagrange multiplier field. Indeed, upon varying (2.17) with respect to $h_{\alpha(s) \dot{\alpha}(s)}^{\mathrm{D}}$ one obtains the Bianchi identity (2.6), and its general solution is given by eq. (2.1), for some real field $h_{\alpha(s) \dot{\alpha}(s)}$. As a result the second term in (2.17) becomes a total derivative, and we end up with the original action $S^{(s)}[\mathcal{C}, \overline{\mathcal{C}}]$. Alternatively, if we first vary (2.17) with respect to $\mathcal{C}^{\alpha(2 s)}$, the equation of motion is

$$
\mathcal{M}_{\alpha(2 s)}=-\mathcal{C}_{\alpha(2 s)}^{\mathrm{D}}
$$

which we may solve to express $\mathcal{C}_{\alpha(2 s)}$ as a function of $\mathcal{C}_{\alpha(2 s)}^{\mathrm{D}}$ and its conjugate. Inserting this solution into (2.17), we obtain the dual model 


$$
\begin{aligned}
& S_{\mathrm{D}}^{(s)}\left[\mathcal{C}^{\mathrm{D}}, \overline{\mathcal{C}}^{\mathrm{D}}\right] \\
& :=\left.\left[S^{(s)}[\mathcal{C}, \overline{\mathcal{C}}]+\int \mathrm{d}^{4} x e\left(\frac{\mathrm{i}}{2} \mathcal{C}^{\alpha(2 s)} \mathcal{C}_{\alpha(2 s)}^{\mathrm{D}}+\text { c.c. }\right)\right]\right|_{\mathcal{C}=\mathcal{C}\left(\mathcal{C}^{\mathrm{D}}, \overline{\mathcal{C}}^{\mathrm{D}}\right)} .
\end{aligned}
$$

Now, given an action $S^{(s)}[\mathcal{C}, \overline{\mathcal{C}}]$ satisfying (2.12), our aim is to show that it satisfies

$$
S_{\mathrm{D}}^{(s)}[\mathcal{C}, \overline{\mathcal{C}}]=S^{(s)}[\mathcal{C}, \overline{\mathcal{C}}]
$$

which means that the corresponding Lagrangian is invariant under Legendre transformations. A routine calculation allows one to show that the following functional

$$
S^{(s)}[\mathcal{C}, \overline{\mathcal{C}}]+\int \mathrm{d}^{4} x e\left(\frac{\mathrm{i}}{4} \mathcal{C}^{\alpha(2 s)} \mathcal{C}_{\alpha(2 s)}+\text { c.c. }\right)
$$

is invariant under (2.10). The latter may be exponentiated to obtain the finite $\mathrm{U}(1)$ duality transformations

$$
\begin{gathered}
\mathcal{C}_{\alpha(2 s)}^{\prime}=\cos \lambda \mathcal{C}_{\alpha(2 s)}+\sin \lambda \mathcal{M}_{\alpha(2 s)} \\
\mathcal{M}_{\alpha(2 s)}^{\prime}=-\sin \lambda \mathcal{C}_{\alpha(2 s)}+\cos \lambda \mathcal{M}_{\alpha(2 s)}
\end{gathered}
$$

Performing such a transformation with $\lambda=\frac{\pi}{2}$ on (2.22) yields

$S^{(s)}[\mathcal{C}, \overline{\mathcal{C}}]=S^{(s)}\left[\mathcal{C}^{\mathrm{D}}, \overline{\mathcal{C}}^{\mathrm{D}}\right]-\int \mathrm{d}^{4} x e\left(\frac{\mathrm{i}}{2} \mathcal{C}^{\alpha(2 s)} \mathcal{C}_{\alpha(2 s)}^{\mathrm{D}}+\right.$ c.c. $)$

Upon inserting this expression into (2.20), we obtain (2.21).

In the above analysis, we made use of the fact that the general solution of the Bianchi identity (2.6) is given by (2.1). To justify this claim, it suffices to work in Minkowski space. Let $\mathcal{C}_{\alpha(2 s)}$ be a field subject to Eq. (2.6), with $\nabla_{a}=\partial_{a}$. Introduce its descendant defined by

$$
\left.h_{\alpha(s) \dot{\alpha}(s)}^{\perp}:=\partial_{\dot{\alpha}_{1}}^{\beta_{1}} \ldots \partial^{\beta_{s}} \dot{\alpha}_{s} \mathcal{C}_{\alpha(s) \beta(s)}=\partial^{\beta_{1}}{ }_{\left(\dot{\alpha}_{1}\right.} \ldots \partial^{\beta_{s}} \dot{\alpha}_{s}\right) \mathcal{C}_{\alpha(s) \beta(s)} \text {, }
$$

which is automatically transverse,

$$
\partial^{\beta \dot{\beta}} h_{\beta \alpha(s-1) \dot{\beta} \dot{\alpha}(s-1)}^{\perp}=0
$$

The Bianchi identity (2.6) tells us that $h_{\alpha(s) \dot{\alpha}(s)}^{\perp}$ is real, $\overline{h_{\alpha(s) \dot{\alpha}(s)}^{\perp}}=h_{\alpha(s) \dot{\alpha}(s)}^{\perp}$. Now we can express $\mathcal{C}_{\alpha(2 s)}$ in terms of (2.25a),

$$
\begin{aligned}
\mathcal{C}_{\alpha(2 s)} & =\square^{-s} \partial_{\left(\alpha_{1}\right.} \dot{\beta}_{1} \ldots \partial_{\alpha_{s}} \dot{\beta}_{s} h_{\left.\alpha_{s+1} \ldots \alpha_{2 s}\right) \dot{\beta}(s)}^{\perp} \\
& =\partial_{\left(\alpha_{1}\right.} \dot{\beta}_{1} \ldots \partial_{\alpha_{s}} \dot{\beta}_{s} h_{\left.\alpha_{s+1} \ldots \alpha_{2 s}\right) \dot{\beta}(s)},
\end{aligned}
$$

where $\square=\partial^{a} \partial_{a}$. In the final relation the real field $h_{\alpha(s) \dot{\alpha}(s)}$ is not assumed to be transverse. This field is related to $\square^{-s} h_{\alpha(s) \dot{\alpha}(s)}^{\perp}$ by a gauge transformation

$$
\delta_{\zeta} h_{\alpha(s) \dot{\alpha}(s)}=\partial_{\left(\alpha _ { 1 } \left(\dot{\alpha}_{1}\right.\right.} \zeta_{\left.\left.\alpha_{2} \ldots \alpha_{s}\right) \dot{\alpha}_{2} \ldots \dot{\alpha}_{s}\right)},
$$

with a real gauge parameter $\zeta_{\alpha(s-1) \dot{\alpha}(s-1)}$. Our consideration may be extended to the $\mathcal{N}=1$ and $\mathcal{N}=2$ supersymmetric cases studied in the next sections.

\section{Auxiliary variable formulation}

As a generalization of the Ivanov-Zupnik [6-8] approach, here we will introduce a powerful formalism to generate duality-invariant models that makes use of auxiliary variables.

Consider the following action functional

$$
\begin{aligned}
S^{(s)}[\mathcal{C}, \overline{\mathcal{C}}, \rho, \bar{\rho}]= & (-1)^{s} \int \mathrm{d}^{4} x e\left\{2 \rho \mathcal{C}-\rho^{2}-\frac{1}{2} \mathcal{C}^{2}\right\} \\
& + \text { c.c. }+\mathcal{S}_{\text {int }}^{(s)}[\rho, \bar{\rho}] .
\end{aligned}
$$

Here we have introduced the auxiliary variable $\rho_{\alpha(2 s)}$ which is chosen to be an unconstrained primary dimension-2 field,

$$
K_{b} \rho_{\alpha(2 s)}=0, \quad \mathbb{D} \rho_{\alpha(2 s)}=2 \rho_{\alpha(2 s)}
$$

The functional $\mathcal{S}_{\text {int }}^{(s)}[\rho, \bar{\rho}]$, by definition, contains cubic and higher powers of $\rho_{\alpha(2 s)}$ and its conjugate. The equation of motion for $\rho^{\alpha(2 s)}$ is

$$
\rho_{\alpha(2 s)}=\mathcal{C}_{\alpha(2 s)}+\frac{(-1)^{s}}{2} \frac{\delta \mathcal{S}_{\text {int }}^{(s)}[\rho, \bar{\rho}]}{\delta \rho^{\alpha(2 s)}}
$$

Equation (2.30) allows one to express $\rho_{\alpha(2 s)}$ as a functional of $\mathcal{C}_{\alpha(2 s)}$ and its conjugate. This means that (2.28) is equivalent to a CHS theory with action

$S^{(s)}[\mathcal{C}, \overline{\mathcal{C}}]=\frac{(-1)^{s}}{2} \int \mathrm{d}^{4} x e \mathcal{C}^{2}+$ c.c. $+S_{\text {int }}^{(s)}[\mathcal{C}, \overline{\mathcal{C}}]$

Thus, (2.28) and (2.31) provide two equivalent realizations of the same model.

The power of this formulation is most evident when the self-duality equation (2.12) is applied. A routine computation reveals that this constraint is equivalent to

$$
\operatorname{Im} \int \mathrm{d}^{4} x e \rho^{\alpha(2 s)} \frac{\delta \mathcal{S}_{\text {int }}^{(s)}[\rho, \bar{\rho}]}{\delta \rho^{\alpha(2 s)}}=0
$$


Thus, self-duality of the action (2.28) is equivalent to the requirement that $\mathcal{S}_{\mathrm{int}}^{(s)}[\rho, \bar{\rho}]$ is invariant under rigid $\mathrm{U}(1)$ phase transformations

$$
\mathcal{S}_{\text {int }}^{(s)}\left[\mathrm{e}^{\mathrm{i} \varphi} \rho, \mathrm{e}^{-i \varphi} \bar{\rho}\right]=\mathcal{S}_{\text {int }}^{(s)}[\rho, \bar{\rho}], \quad \varphi \in \mathbb{R}
$$

For instance we can consider the model

$$
\mathcal{S}_{\text {int }}^{(s)}[\rho, \bar{\rho} ; \Psi]=\int \mathrm{d}^{4} x e \Psi^{4} \mathfrak{F}\left(\frac{\rho^{2} \bar{\rho}^{2}}{\Psi^{8}}\right),
$$

where $\mathfrak{F}(x)$ is a real analytic function of a real variable. However, such models are not conformal if the action does depend on $\Psi$. The condition of conformal invariance imposes additional nontrivial restrictions.

\section{Conformal U(1) duality-invariant models}

In the $s=1$ case, there is the unique conformal U(1) duality-invariant electrodynamics proposed in [29] (see also [30]). It was called ModMax electrodynamics in [29]. It turns out that for $s>1$, families of conformal $\mathrm{U}(1)$ duality-invariant models exist.

As a warm-up example, let us consider the following nonlinear conformal action:

$$
\begin{aligned}
S^{(s)}[\mathcal{C}, \overline{\mathcal{C}}]= & \frac{(-1)^{s} \alpha}{2} \int \mathrm{d}^{4} x e\left\{\mathcal{C}^{2}+\overline{\mathcal{C}}^{2}\right\} \\
& +\beta \int \mathrm{d}^{4} x e \sqrt{\mathcal{C}^{2} \overline{\mathcal{C}}^{2}}, \quad \alpha, \beta \in \mathbb{R}
\end{aligned}
$$

Requiring this action to obey the self-duality equation (2.12), we obtain the constraint

$$
\alpha^{2}-\beta^{2}=1 \Rightarrow \alpha=\cosh \gamma, \quad \beta=\sinh \gamma, \quad \gamma \in \mathbb{R} .
$$

Thus, the nonlinear theory

$$
\begin{aligned}
S^{(s)}[\mathcal{C}, \overline{\mathcal{C}}]= & \frac{(-1)^{s} \cosh \gamma}{2} \int \mathrm{d}^{4} x e\left\{\mathcal{C}^{2}+\overline{\mathcal{C}}^{2}\right\} \\
& +\sinh \gamma \int \mathrm{d}^{4} x e \sqrt{\mathcal{C}^{2} \overline{\mathcal{C}}^{2}}, \quad \gamma \in \mathbb{R},
\end{aligned}
$$

is a one-parameter conformal $\mathrm{U}(1)$ duality-invariant extension of the free CHS action (E1). In the $s=1$ case our model coincides with ModMax electrodynamics.

In order to construct more general models, it is advantageous to make use of the auxiliary variable formulation described above. We introduce algebraic invariants of the symmetric rank- $(2 s)$ spinor $\rho_{\alpha(2 s)}$, which has the same algebraic properties as the linearized spin- $s$ Weyl tensor $\mathcal{C}_{\alpha(2 s)}$ :

$$
\begin{aligned}
\rho^{2} & :=(-1)^{s} \rho_{\alpha(s)}^{\beta(s)} \rho_{\beta(s)}^{\alpha(s)}, \\
\rho^{3} & :=\rho_{\alpha(s)}{ }^{\beta(s)} \rho_{\beta(s)^{\gamma(s)}} \rho_{\gamma(s)}^{\alpha(s)}, \ldots
\end{aligned}
$$

If $s$ is odd, all invariants $\rho^{2 n+1}$, with $n$ a non-negative integer, vanish.

For simplicity, we restrict our analysis to the conformal graviton, $s=2$. In this case the family of invariants (2.38) contains only two functionally independent invariants [42], $\rho^{2}$ and $\rho^{3}$. In particular, one may show that

$$
s=2: \quad \rho^{4}=\frac{1}{2}\left(\rho^{2}\right)^{2} .
$$

Now we choose the self-interaction in (2.28) to be of the form

$$
\mathcal{S}_{\text {int }}^{(2)}[\rho, \bar{\rho}]=\int \mathrm{d}^{4} x e\left\{\beta\left(\rho^{2} \bar{\rho}^{2}\right)^{\frac{1}{2}}+\kappa\left(\rho^{3} \bar{\rho}^{3}\right)^{\frac{1}{3}}\right\}
$$

where $\beta$ and $\kappa$ are real coupling constants. The resulting model is clearly conformal and $\mathrm{U}(1)$ duality invariant. For $\kappa \neq 0$, elimination of the auxiliary variables $\rho_{\alpha(4)}$ and $\bar{\rho}_{\dot{\alpha}(4)}$ does not result in a simple action like (2.37). In particular, such an elimination, to quadratic order in the couplings, yields the following self-dual model:

$$
\begin{aligned}
& S^{(2)}[\mathcal{C}, \overline{\mathcal{C}}]=\int \mathrm{d}^{4} x e\left\{\frac{1}{2}\left(1+\frac{1}{2} \beta^{2}\right)\left(\mathcal{C}^{2}+\overline{\mathcal{C}}^{2}\right)\right. \\
& +\beta\left(\mathcal{C}^{2} \overline{\mathcal{C}}^{2}\right)^{\frac{1}{2}}+\kappa\left(\mathcal{C}^{3} \overline{\mathcal{C}}^{3}\right)^{\frac{1}{3}} \\
& +\frac{1}{2} \beta \kappa \frac{\left(\mathcal{C}^{3}\right)^{2} \overline{\mathcal{C}}^{2}+\left(\overline{\mathcal{C}}^{3}\right)^{2} \mathcal{C}^{2}}{\left(\mathcal{C}^{3} \overline{\mathcal{C}}^{3}\right)^{\frac{2}{3}}\left(\mathcal{C}^{2} \overline{\mathcal{C}}^{2}\right)^{\frac{1}{2}}}+\frac{1}{12} \kappa^{2} \frac{\left(\mathcal{C}^{2}\right)^{2}+\left(\overline{\mathcal{C}}^{2}\right)^{2}}{\left(\mathcal{C}^{3} \overline{\mathcal{C}}^{3}\right)^{\frac{1}{3}}} \\
& \left.-\frac{1}{24} \kappa^{2} \frac{\left(\mathcal{C}^{3}\right)^{2}\left(\overline{\mathcal{C}}^{2}\right)^{2}+\left(\overline{\mathcal{C}}^{3}\right)^{2}\left(\mathcal{C}^{2}\right)^{2}}{\left(\mathcal{C}^{3} \overline{\mathcal{C}}^{3}\right)^{\frac{4}{3}}}+\ldots\right\} .
\end{aligned}
$$

The ellipsis in (2.41) denotes additional contributions to the full nonlinear theory which are cubic or higher order in the coupling constants. We emphasize that for the special case $\kappa=0$ the above action yields (2.37). A proof of this result is given in Appendix D.

For $s>2$ the number of algebraic invariants of $\rho_{\alpha(2 s)}$ grows, and therefore one can define families of conformal $\mathrm{U}(1)$ duality-invariant models.

\section{III. $\mathcal{N}=1$ DUALITY-INVARIANT SCHS MODELS}

The purely bosonic study undertaken in the previous section can be generalized to the supersymmetric case. To this end, we consider a dynamical system describing the propagation of a conformal superspin- $\left(s+\frac{1}{2}\right)$ gauge multiplet $H_{\alpha(s) \dot{\alpha}(s)}, s>0$, in $\mathcal{N}=1$ curved superspace [35,43]. 
The prepotential $H_{\alpha(s) \dot{\alpha}(s)}$ is a real unconstrained superfield being defined modulo the gauge transformations

$$
\delta_{\zeta} H_{\alpha(s) \dot{\alpha}(s)}=\nabla_{\left(\alpha_{1}\right.} \bar{\zeta}_{\left.\alpha_{2} \ldots \alpha_{s}\right) \dot{\alpha}(s)}-\bar{\nabla}_{\left(\dot{\alpha}_{1}\right.} \zeta_{\left.\alpha(s) \dot{\alpha}_{2} \ldots \dot{\alpha}_{s}\right)},
$$

with a primary unconstrained gauge parameter $\zeta_{\alpha(s) \dot{\alpha}(s-1)}$. This gauge transformation law is superconformal provided

$$
K^{B} H_{\alpha(s) \dot{\alpha}(s)}=0, \quad \mathbb{D} H_{\alpha(s) \dot{\alpha}(s)}=-s H_{\alpha(s) \dot{\alpha}(s)}
$$

where $K^{B}=\left(K^{b}, S^{\beta}, \bar{S}_{\dot{\beta}}\right)$ are the special conformal generators, see Appendix $B$. The action functional of the dynamical system is required to take the form $S^{(s)}[\mathcal{W}, \overline{\mathcal{W}}]$, where $\mathcal{W}_{\alpha(2 s+1)}$ is the following chiral descendant of $H_{\alpha(s) \dot{\alpha}(s)}$ :

$$
\begin{aligned}
\mathcal{W}_{\alpha(2 s+1)} & =-\frac{1}{4} \bar{\nabla}^{2} \nabla_{\left(\alpha_{1}\right.} \dot{\beta}_{1} \ldots \nabla_{\alpha_{s}} \dot{\beta}_{s} \nabla_{\alpha_{s+1}} H_{\left.\alpha_{s+2} \ldots \alpha_{2 s+1}\right) \dot{\beta}(s)}, \\
\bar{\nabla}^{\dot{\beta}} \mathcal{W}_{\alpha(2 s+1)} & =0
\end{aligned}
$$

This field strength proves to be primary in a generic supergravity background [35,43],

$$
K^{B} \mathcal{W}_{\alpha(2 s+1)}=0, \quad \mathbb{D} \mathcal{W}_{\alpha(2 s+1)}=\frac{3}{2} \mathcal{W}_{\alpha(2 s+1)}
$$

However, the gauge transformations (3.1) leave $\mathcal{W}_{\alpha(2 s+1)}$ invariant only if the supergravity background is conformally flat,

$$
W_{\alpha(3)}=0 \Rightarrow \delta_{\zeta} \mathcal{W}_{\alpha(2 s+1)}=0,
$$

where $W_{\alpha(3)}$ is the background super-Weyl tensor, see Appendix B. For the remainder of this section we will assume such a geometry.

It should be pointed out that the prepotential $H_{\alpha \dot{\alpha}}$ encodes the linearized conformal supergravity multiplet and its field strength $\mathcal{W}_{\alpha(3)}$ is the linearized super-Weyl tensor $[44,45]$. We will refer to $\mathcal{W}_{\alpha(2 s+1)}$ for $s>1$ as the linearized superspin- $\left(s+\frac{1}{2}\right)$ Weyl tensor, or simply as a higher-spin super-Weyl tensor.

With the exception of the gauge transformation law (3.1), the above results are also valid in the $s=0$ case corresponding to the massless vector multiplet. As is well known, its prepotential $H$ is defined modulo the gauge transformations [46,47]

$$
\delta_{\chi} H=\chi+\bar{\chi}, \quad \bar{\nabla}^{\dot{\beta}} \chi=0,
$$

with the chiral scalar $\chi$ being primary and dimensionless. Unlike the $s \geq 1$ case considered above, the vector multiplet field strength $\mathcal{W}_{\alpha}=-\frac{1}{4} \bar{\nabla}^{2} \nabla_{\alpha} H$ is gauge invariant, $\delta_{\chi} \mathcal{W}_{\alpha}=0$, for an arbitrary supergravity background.

\section{A. U(1) duality-invariant models}

It is important to note that the field strength (3.3) obeys the Bianchi identity ${ }^{4}$ [48]

$$
\begin{aligned}
& \left.\nabla^{\beta_{1}}{ }_{\left(\dot{\alpha}_{1}\right.} \ldots \nabla^{\beta_{s}} \dot{\alpha}_{s}\right) \nabla^{\beta_{s+1}} \mathcal{W}_{\alpha(s) \beta(s+1)} \\
& \quad=-\nabla_{\left(\alpha_{1}\right.} \dot{\beta}_{1} \ldots \nabla_{\left.\alpha_{s}\right)} \dot{\beta}_{s} \bar{\nabla}^{\dot{\beta}_{s+1}} \overline{\mathcal{W}}_{\dot{\alpha}(s) \dot{\beta}(s+1)} .
\end{aligned}
$$

Moreover, the real superfield

$$
\mathcal{B}_{\alpha(s) \dot{\alpha}(s)}:=\nabla_{\left(\dot{\alpha}_{1} \ldots \nabla^{\beta_{s}} \dot{\alpha}_{s}\right)}^{\beta_{s+1}} \mathcal{W}_{\alpha(s) \beta(s+1)}
$$

proves to be primary,

$K^{B} \mathcal{B}_{\alpha(s) \dot{\alpha}(s)}=0, \quad \mathbb{D} \mathcal{B}_{\alpha(s) \dot{\alpha}(s)}=(2+s) \mathcal{B}_{\alpha(s) \dot{\alpha}(s)}$,

and may be called the linearized superspin- $\left(s+\frac{1}{2}\right)$ Bach tensor. For $s>1 \mathcal{B}_{\alpha(s) \dot{\alpha}(s)}$ is also referred to as a higher-spin super-Bach tensor.

We assume that $S^{(s)}[\mathcal{W}, \overline{\mathcal{W}}]$ is consistently defined as a functional of a general chiral superfield $\mathcal{W}_{\alpha(2 s+1)}$ and its conjugate. This allows us to introduce the symmetric spinor

$$
\mathrm{i} \mathcal{M}_{\alpha(2 s+1)}:=2 \frac{\delta S^{(s)}[\mathcal{W}, \overline{\mathcal{W}}]}{\delta \mathcal{W}^{\alpha(2 s+1)}}
$$

where the variational derivative is defined by

$$
\delta S^{(s)}[\mathcal{W}, \overline{\mathcal{W}}]=\int \mathrm{d}^{4} x \mathrm{~d}^{2} \theta \mathcal{E} \delta \mathcal{W}^{\alpha(2 s+1)} \frac{\delta S^{(s)}[\mathcal{W}, \overline{\mathcal{W}}]}{\delta \mathcal{W}^{\alpha(2 s+1)}}+\text { c.c. }
$$

and $\mathcal{E}$ is the chiral integration measure. It follows that $\mathcal{M}_{\alpha(2 s+1)}$ is a primary chiral superfield,

$$
\begin{aligned}
K^{B} \mathcal{M}_{\alpha(2 s+1)} & =0, \quad \bar{\nabla}^{\dot{\beta}} \mathcal{M}_{\alpha(2 s+1)}=0, \\
\mathbb{D} \mathcal{M}_{\alpha(2 s+1)} & =\frac{3}{2} \mathcal{M}_{\alpha(2 s+1)} .
\end{aligned}
$$

Varying the action with respect to the prepotential $H_{\alpha(s) \dot{\alpha}(s)}$ yields the equation of motion

$$
\begin{aligned}
& \left.\nabla^{\beta_{1}}{ }_{\left(\dot{\alpha}_{1}\right.} \ldots \nabla^{\beta_{s}} \dot{\alpha}_{s}\right) \nabla^{\beta_{s+1}} \mathcal{M}_{\alpha(s) \beta(s+1)} \\
& \quad=-\nabla_{\left(\alpha_{1}\right.} \dot{\beta}_{1} \ldots \nabla_{\left.\alpha_{s}\right)} \dot{\beta}_{s} \bar{\nabla}^{\dot{\beta}_{s+1}} \overline{\mathcal{M}}_{\dot{\alpha}(s) \dot{\beta}(s+1)} .
\end{aligned}
$$

Here the real superfield $\left.\nabla^{\beta_{1}} \dot{\alpha}_{1} \ldots \nabla^{\beta_{s}} \dot{\alpha}_{s}\right) \nabla^{\beta_{s+1}} \mathcal{M}_{\alpha(s) \beta(s+1)}$ proves to be primary.

The analysis above indicates that the functional form of (3.13) coincides with that of (3.7). It then follows that the

\footnotetext{
${ }^{4}$ This differs from the Bianchi identity for the nonlinear superWeyl tensor (B3b) on historical grounds.
} 
system of equations (3.7) and (3.13) is invariant under the $\mathrm{U}(1)$ duality rotations:

$$
\delta_{\lambda} \mathcal{W}_{\alpha(2 s+1)}=\lambda \mathcal{M}_{\alpha(2 s+1)}, \quad \delta_{\lambda} \mathcal{M}_{\alpha(2 s+1)}=-\lambda \mathcal{W}_{\alpha(2 s+1)},
$$

where $\lambda$ is a constant, real parameter. One may then obtain two equivalent expressions for the variation of $S^{(s)}[\mathcal{W}, \overline{\mathcal{W}}]$ with respect to (3.14)

$$
\begin{aligned}
\delta_{\lambda} S^{(s)}[\mathcal{W}, \overline{\mathcal{W}}] & =\frac{\mathrm{i} \lambda}{4} \int \mathrm{d}^{4} x \mathrm{~d}^{2} \theta \mathcal{E}\left\{\mathcal{W}^{2}-\mathcal{M}^{2}\right\}+\text { c.c. } \\
& =-\frac{\mathrm{i} \lambda}{2} \int \mathrm{d}^{4} x \mathrm{~d}^{2} \theta \mathcal{E} \mathcal{M}^{2}+\text { c.c. }
\end{aligned}
$$

as a generalization of similar derivations in nonlinear $\mathcal{N}=1$ supersymmetric electrodynamics $[9-11] .^{5}$ This implies the self-duality equation

$$
\begin{aligned}
& \operatorname{Im} \int \mathrm{d}^{4} x \mathrm{~d}^{2} \theta \mathcal{E}\left\{\mathcal{W}^{\alpha(2 s+1)} \mathcal{W}_{\alpha(2 s+1)}\right. \\
& \left.+\mathcal{M}^{\alpha(2 s+1)} \mathcal{W}_{\alpha(2 s+1)}\right\}=0
\end{aligned}
$$

which must hold for a general chiral superfield $\mathcal{W}_{\alpha(2 s+1)}$ and its conjugate. Every solution $S^{(s)}[\mathcal{W}, \overline{\mathcal{W}}]$ of the self-duality equation describes a $\mathrm{U}(1)$ duality-invariant theory. In the $s=0$ case, Eq. (3.16) was originally derived in [9] in Minkowski superspace and extended to supergravity in [11]. The simplest solution of the self-duality equation (3.16) is the free $\mathcal{N}=1$ SCHS model (E5), which was introduced in [43] for the cases of Minkowski and anti-de Sitter (AdS) backgrounds and later was generalized to arbitrary conformally flat backgrounds in [35].

The above results allow one to prove, in complete analogy with the nonsupersymmetric analysis conducted in Sec. II B, that the U(1) duality-invariant theories are selfdual under Legendre transformations.

\section{B. Auxiliary variable formulation}

As a generalization of the auxiliary variable formalism sketched in Sec. II C, here we will develop a reformulation of the $\mathrm{U}(1)$ duality-invariant systems introduced in the previous subsection. In the $s=0$ case, it will reduce to the auxiliary superfield approach for U(1) duality-invariant supersymmetric electrodynamics introduced in [26,27].

\footnotetext{
${ }^{5}$ In Eq. (3.15) and the remainder of this section we make use of the notational shorthand $\mathcal{W}^{2}=\mathcal{W}^{\alpha(2 s+1)} \mathcal{W}_{\alpha(2 s+1)}$ and $\overline{\mathcal{W}}^{2}=\overline{\mathcal{W}}_{\dot{\alpha}(2 s+1)} \overline{\mathcal{W}}^{\dot{\alpha}(2 s+1)}$.
}

Consider the action functional

$$
\begin{aligned}
S^{(s)}[\mathcal{W}, \overline{\mathcal{W}}, \eta, \bar{\eta}]= & (-1)^{s} \int \mathrm{d}^{4} x \mathrm{~d}^{2} \theta \mathcal{E}\left\{\eta \mathcal{W}-\frac{1}{2} \eta^{2}-\frac{1}{4} \mathcal{W}^{2}\right\} \\
& + \text { c.c. }+\mathcal{S}_{\text {int }}^{(s)}[\eta, \bar{\eta}] .
\end{aligned}
$$

Here we have introduced the new dimension-3/2 multiplet $\eta_{\alpha(2 s+1)}$, which is required to be primary and covariantly chiral,

$$
K^{B} \eta_{\alpha(2 s+1)}=0, \quad \bar{\nabla}_{\dot{\alpha}} \eta_{\alpha(2 s+1)}=0, \quad \mathbb{D} \eta_{\alpha(2 s+1)}=\frac{3}{2} \eta_{\alpha(2 s+1)} .
$$

By definition, the functional $\mathcal{S}_{\text {int }}^{(s)}[\eta, \bar{\eta}]$, contains cubic and higher powers of $\eta_{\alpha(2 s+1)}$ and its conjugate.

The equation of motion for $\eta^{\alpha(2 s+1)}$ is

$$
\eta_{\alpha(2 s+1)}=\mathcal{W}_{\alpha(2 s+1)}+(-1)^{s} \frac{\delta \mathcal{S}_{\text {int }}^{(s)}[\eta, \bar{\eta}]}{\delta \eta^{\alpha(2 s+1)}} .
$$

Employing perturbation theory, Eq. (3.19) allows one to express $\eta_{\alpha(2 s+1)}$ as a functional of $\mathcal{W}_{\alpha(2 s+1)}$ and its conjugate. This means that (3.17) is dual to a SCHS theory with action

$$
S^{(s)}[\mathcal{W}, \overline{\mathcal{W}}]=\frac{(-1)^{s}}{4} \int \mathrm{d}^{4} x \mathrm{~d}^{2} \theta \mathcal{E} \mathcal{W}^{2}+\text { c.c. }+S_{\text {int }}^{(s)}[\mathcal{W}, \overline{\mathcal{W}}]
$$

Thus, (3.17) and (3.20) provide two equivalent realizations of the same model.

The power of this formulation is most evident when the self duality equation (3.16) is applied. A routine computation reveals that this constraint is equivalent to

$$
\operatorname{Im} \int \mathrm{d}^{4} x \mathrm{~d}^{2} \theta \mathcal{E} \eta^{\alpha(2 s+1)} \frac{\delta \mathcal{S}_{\text {int }}^{(s)}[\eta, \bar{\eta}]}{\delta \eta^{\alpha(2 s+1)}}=0 .
$$

Thus, self-duality of the action (3.17) is equivalent to the requirement that $\mathcal{S}_{\mathrm{int}}^{(s)}[\eta, \bar{\eta}]$ is invariant under rigid $\mathrm{U}(1)$ phase transformations

$$
\mathcal{S}_{\text {int }}^{(s)}\left[\mathrm{e}^{\mathrm{i} \varphi} \eta, \mathrm{e}^{-i \varphi} \bar{\eta}\right]=\mathcal{S}_{\text {int }}^{(s)}[\eta, \bar{\eta}], \quad \varphi \in \mathbb{R} .
$$

Within the superconformal approach to supergravitymatter dynamical systems [39], every theory of Einstein supergravity coupled to supersymmetric matter is realized as a coupling of the same matter multiplets to conformal supergravity and a superconformal compensator, see, e.g., [49]. Truly superconformal theories are independent of any compensator. Different off-shell formulations for 
Einstein supergravity correspond to different choices of the compensating multiplet. The most famous compensators are (i) a chiral compensator $S_{0}$ of the old minimal supergravity and (ii) a real linear compensator $\mathbb{L}=\overline{\mathbb{L}}$ of the new minimal supergravity. Both $S_{0}$ and $\mathbb{L}$ are required to be nowhere vanishing, such that $\left(S_{0}\right)^{-1}$ and $\mathbb{L}^{-1}$ exist, and they satisfy the following superconformal properties:

$$
\begin{aligned}
& K^{B} S_{0}=0, \quad \bar{\nabla}_{\dot{\alpha}} S_{0}=0, \quad \mathbb{D} S_{0}=S_{0} ; \\
& K^{B} \mathbb{L}=0, \quad \bar{\nabla}^{2} \mathbb{L}=0 \Rightarrow \mathbb{D} \mathbb{L}=2 \mathbb{L} .
\end{aligned}
$$

If we allow the action (3.20) to depend on a superconformal compensator, either $S_{0}$ or $\mathbb{L}$, we can generate families of $U(1)$ duality-invariant theories which satisfy the condition (3.22). For simplicity, unless otherwise stated, here we will restrict our attention to the following interaction:

$$
\begin{aligned}
\mathcal{S}_{\text {int }}^{(s)}[\eta, \bar{\eta} ; \Upsilon]= & \frac{1}{2(2 s+2) !} \int \mathrm{d}^{4} x \mathrm{~d}^{2} \theta \mathrm{d}^{2} \bar{\theta} E \frac{\eta^{2 s+2} \bar{\eta}^{2 s+2}}{\Upsilon^{3 s+2}} \\
& \times \mathfrak{F}^{(s)}(v, \bar{v}),
\end{aligned}
$$

where

$$
v:=\frac{1}{8} \nabla^{2}\left[\eta^{2} \Upsilon^{-2}\right],
$$

and the compensator $\Upsilon$ has one of the two realizations: either $\Upsilon=S_{0} \bar{S}_{0}$ or $\Upsilon=\mathbb{L}$. Applying (3.22), we find that our model is $\mathrm{U}(1)$ duality invariant provided

$$
\mathfrak{F}^{(s)}(v, \bar{v})=\mathfrak{F}^{(s)}(v \bar{v}) .
$$

\section{Superconformal U(1) duality-invariant model}

The U(1) duality-invariant model (3.24) is superconformal if the action is independent of $\Upsilon$. This uniquely fixes the functional form of $\mathfrak{F}(v \bar{v})$ modulo a single real parameter

$$
\mathfrak{\mho}_{\mathrm{SC}}^{(s)}(v \bar{v})=\frac{\kappa}{(v \bar{v})^{\frac{1}{4}(3 s+2)}}, \quad \kappa \in \mathbb{R} .
$$

Employing (3.19), we arrive at

$$
\begin{aligned}
\mathcal{W}_{\alpha(2 s+1)}= & \eta_{\alpha(2 s+1)}\left\{1+\frac{(-1)^{s} \kappa}{8(2 s+2) !} \bar{\nabla}^{2}\right. \\
& \times\left[(2 s+2) \frac{\eta^{2 s} \bar{\eta}^{2 s+2}}{\Upsilon^{3 s+2}} \widetilde{\mho}_{\mathrm{SC}}^{(s)}\right. \\
& \left.\left.+\frac{\bar{\eta}^{2 s+2}}{4 \Upsilon^{3 s+2}} \nabla^{2}\left(\eta^{2 s+2} \partial_{v} \widetilde{\mho}_{\mathrm{SC}}^{(s)}\right)\right]\right\},
\end{aligned}
$$

which, along with its conjugate, allows us to integrate out the auxiliary variables present in (3.17). The final result for $s=0$ is the model for superconformal U(1) dualityinvariant electrodynamics introduced in [28,31]

$$
\begin{aligned}
S^{(0)}[\mathcal{W}, \overline{\mathcal{W}}]= & \frac{1}{4} \cosh \gamma \int \mathrm{d}^{4} x \mathrm{~d}^{2} \theta \mathcal{E} \mathcal{W}^{2}+\text { c.c. } \\
& +\frac{1}{4} \sinh \gamma \int \mathrm{d}^{4} x \mathrm{~d}^{2} \theta \mathrm{d}^{2} \bar{\theta} E \frac{\mathcal{W}^{2} \overline{\mathcal{W}}^{2}}{\Upsilon^{2} \sqrt{u \bar{u}}},
\end{aligned}
$$

where $\gamma$ is a real coupling constant. For $s>0$ the resulting model is

$$
\begin{aligned}
& S^{(s>0)}[\mathcal{W}, \overline{\mathcal{W}}] \\
& =\frac{(-1)^{s}}{4} \int \mathrm{d}^{4} x \mathrm{~d}^{2} \theta \mathcal{E} \mathcal{W}^{2}+\text { c.c. } \\
& \quad+\frac{\kappa}{2(2 s+2) !} \int \mathrm{d}^{4} x \mathrm{~d}^{2} \theta \mathrm{d}^{2} \bar{\theta} E \frac{\left(\mathcal{W}^{2} \overline{\mathcal{W}}^{2}\right)^{s+1}}{\Upsilon^{3 s+2}(u \bar{u})^{\frac{1}{4}(3 s+2)}}
\end{aligned}
$$

In both cases we have made use of the shorthand

$$
u=\frac{1}{8} \nabla^{2}\left[\frac{\mathcal{W}^{2}}{\Upsilon^{2}}\right]
$$

We note that (3.28) is invariant under the following rescaling of the conformal compensator:

$$
\Upsilon^{\prime}=\mathrm{e}^{2 \sigma} \Upsilon
$$

which implies that the dependence of (3.28) on $\Upsilon$ is purely superficial. Consequently, the action is superconformal.

It is important to note that, at the component level, the purely bosonic sector of the interaction (the $\kappa$-term) present in (3.28) identically vanishes. ${ }^{6}$ Thus, these actions describe different duality-invariant models than those presented in Sec. II D. However, one may construct a supersymmetric duality-invariant model that contains, for instance, the bosonic theory (2.37) at the component level.

Consider the following supersymmetric duality-invariant model:

$$
\begin{aligned}
S^{(s)}[\mathcal{W}, \overline{\mathcal{W}}, \eta, \bar{\eta} ; \Upsilon] \\
=(-1)^{s} \int \mathrm{d}^{4} x \mathrm{~d}^{2} \theta \mathcal{E}\left\{\eta \mathcal{W}-\frac{1}{2} \eta^{2}-\frac{1}{4} \mathcal{W}^{2}\right\}+\text { c.c. } \\
\quad+\frac{\beta}{8} \int \mathrm{d}^{4} x \mathrm{~d}^{2} \theta \mathrm{d}^{2} \bar{\theta} E \frac{\eta^{2} \bar{\eta}^{2}}{\Upsilon^{2} \sqrt{v \bar{v}}}
\end{aligned}
$$

\footnotetext{
${ }^{6}$ The numerator in the $\kappa$-term in (3.28) contains a product of $4(s+1)$ fermionic superfields. Since the component reduction of this term is computed according to the rule (3.33b), it is clear we do not have enough spinor derivatives to convert all the fermionic superfields into bosonic ones for $s>0$.
} 
It does not enjoy invariance under (3.30) and thus (3.31) is not superconformal. However, we are going to demonstrate below that, at the component level, this model contains certain conformal duality-invariant actions. For this we restrict our attention to the bosonic sector of (3.31). The bosonic field strengths contained in $\mathcal{W}_{\alpha(2 s+1)}$ are

$$
\begin{aligned}
\mathcal{C}_{\alpha(2 s+2)} & =\left.\frac{\sqrt{2} \mathrm{i}}{4} \nabla_{\left(\alpha_{1}\right.} \mathcal{W}_{\left.\alpha_{2} \ldots \alpha_{2 s+2}\right)}\right|_{\theta=0}, \\
\mathcal{C}_{\alpha(2 s)} & =\left.\frac{1}{4} \sqrt{\frac{2 s+1}{s+1}} \nabla^{\beta} \mathcal{W}_{\beta \alpha(2 s)}\right|_{\theta=0} .
\end{aligned}
$$

The bosonic component fields of $\eta_{\alpha(2 s+1)}$ are

$$
\begin{aligned}
\rho_{\alpha(2 s+2)} & =\left.\frac{\sqrt{2} \mathrm{i}}{4} \nabla_{\left(\alpha_{1}\right.} \eta_{\left.\alpha_{2} \ldots \alpha_{2 s+2}\right)}\right|_{\theta=0}, \\
\rho_{\alpha(2 s)} & =\left.\frac{1}{4} \sqrt{\frac{2 s+1}{s+1}} \nabla^{\beta} \eta_{\beta \alpha(2 s)}\right|_{\theta=0} .
\end{aligned}
$$

Action (3.31) can be reduced to components using the standard reduction rules

$$
\int \mathrm{d}^{4} x \mathrm{~d}^{2} \theta \mathcal{E} \mathcal{L}_{c}=-\left.\frac{1}{4} \int \mathrm{d}^{4} x e \nabla^{2} \mathcal{L}_{c}\right|_{\theta=0}, \quad \bar{\nabla}^{\dot{\alpha}} \mathcal{L}_{c}=0,
$$

$$
\int \mathrm{d}^{4} x \mathrm{~d}^{2} \theta \mathrm{d}^{2} \bar{\theta} E \mathcal{L}=\left.\frac{1}{16} \int \mathrm{d}^{4} x e \nabla^{2} \bar{\nabla}^{2} \mathcal{L}\right|_{\theta=0}
$$

Since we are interested only in the bosonic sector, it suffices to approximate

$$
\begin{aligned}
\left.v\right|_{\theta=0} & \approx \frac{2}{(\Upsilon \mid)^{2}}\left\{\rho^{\alpha(2 s+2)} \rho_{\alpha(2 s+2)}-\rho^{\alpha(2 s)} \rho_{\alpha(2 s)}\right\}, \\
\Upsilon \mid & \left.\equiv \Upsilon\right|_{\theta=0} .
\end{aligned}
$$

Further, it may be checked that the interaction (the $\beta$-term) in (3.31) does not contain contributions which are (i) linear in $\rho_{\alpha(2 s)}$ and its conjugate and (ii) linear in $\rho_{\alpha(2 s+2)}$ and its conjugate. Therefore, if we switch off the spin-s field,

$$
\mathcal{C}_{\alpha(2 s)}=0
$$

then

$$
\rho_{\alpha(2 s)}=0
$$

is a solution of the corresponding equation of motion. Under the conditions (3.35) and (3.36), the resulting bosonic action proves to coincide with $S^{(s+1)}[\mathcal{C}, \overline{\mathcal{C}}, \rho, \bar{\rho}]$ obtained from (D1) by replacing $s$ with $s+1$. We emphasize that the compensator $\Upsilon$ does not contribute to this action, and therefore $S^{(s+1)}[\mathcal{C}, \overline{\mathcal{C}}, \rho, \bar{\rho}]$ is locally conformal. ${ }^{7}$ Instead of considering the branch (3.35), we may switch off the spin- $(s+1)$ field, $\mathcal{C}_{\alpha(2 s+2)}=0$. Then it follows that $\rho_{\alpha(2 s+2)}=0$ is a solution of the corresponding equation of motion, and the resulting bosonic action proves to coincide with the conformal duality-invariant action $S^{(s)}[\mathcal{C}, \overline{\mathcal{C}}, \rho, \bar{\rho}]$, Eq. (D1).

To conclude this section, we derive a supersymmetric extension of the cubic interaction present in (2.40). For this purpose, we will fix $s=1$ in (3.17). The relevant interaction is constructed in terms of the two scalar primary descendants of $\eta_{\alpha(3)}$ :

$$
\Xi=\mathrm{i} \eta^{\alpha(2) \beta} \eta^{\alpha(2)} \nabla_{\left(\alpha_{1}\right.} \eta_{\left.\alpha_{2} \alpha_{3} \alpha_{4}\right)}, \quad w=4 \nabla^{2}\left[\frac{\Xi}{\Upsilon^{3}}\right],
$$

and the conformal compensator $\Upsilon$. Using these, we construct the following action:

$$
\begin{aligned}
S^{(1)}[\mathcal{W}, \overline{\mathcal{W}}, \eta, \bar{\eta} ; \Upsilon]= & -\int \mathrm{d}^{4} x \mathrm{~d}^{2} \theta \mathcal{E}\left\{\eta \mathcal{W}-\frac{1}{2} \eta^{2}-\frac{1}{4} \mathcal{W}^{2}\right\} \\
& + \text { c.c. } \\
& +32 \kappa \int \mathrm{d}^{4} x \mathrm{~d}^{2} \theta \mathrm{d}^{2} \bar{\theta} E \frac{\Xi \bar{\Xi}}{\Upsilon^{4}(w \bar{w})^{\frac{2}{3}}} .
\end{aligned}
$$

We note that it is not superconformal as it lacks invariance under (3.30), though we will show that, at the component level, it contains the cubic interaction present in (2.40). To demonstrate this, we restrict our attention to the bosonic sector of (3.38), which allows us to approximate

$$
\begin{aligned}
\left.w\right|_{\theta=0} \approx & -\frac{\sqrt{2}}{4(\Upsilon \mid)^{3}}\left(\rho_{\beta(2)}^{\alpha(2)} \rho_{\gamma(2)}^{\beta(2)} \rho_{\alpha(2)}^{\gamma(2)}\right. \\
& \left.-\frac{9}{32} \rho^{\alpha(2)} \rho^{\alpha(2)} \rho_{\alpha(4)}\right)
\end{aligned}
$$

It follows then that the interaction (the $\kappa$-term) present in (3.38) contains no terms linear in $\rho_{\alpha(2)}$ and its conjugate. Hence, if we switch off the spin-1 field, $\mathcal{C}_{\alpha(2)}=0$, then $\rho_{\alpha(2)}=0$ solves the corresponding equation of motion. Under these conditions, the resulting bosonic action takes the form

$$
\begin{aligned}
S^{(2)}[\mathcal{C}, \overline{\mathcal{C}}, \rho, \bar{\rho}]= & \int \mathrm{d}^{4} x e\left\{2 \rho \mathcal{C}-\rho^{2}-\frac{1}{2} \mathcal{C}^{2}\right\}+\text { c.c. } \\
& +\kappa \int \mathrm{d}^{4} x e\left(\rho^{3} \bar{\rho}^{3}\right)^{\frac{1}{3}}
\end{aligned}
$$

\footnotetext{
${ }^{7}$ As demonstrated in Appendix D, the action (D1) leads to (2.37) upon eliminating the auxiliary field $\rho_{\alpha(2 s)}$ and its conjugate.
} 
In particular, we note that it is locally conformal as it is independent of the compensator. Thus, (3.38) is a supersymmetric extension of the cubic interaction of (2.40). In closing, we emphasize that if one instead attempts to consider the branch $\mathcal{C}_{\alpha(4)}=0$ and $\mathcal{C}_{\alpha(2)} \neq 0$, it is not legal to set $\rho_{\alpha(4)}=0$. Such a choice would imply $\left.w\right|_{\theta=0} \approx 0$, making the corresponding component action of (3.38) undefined.

\section{IV. $\mathcal{N}=2$ DUALITY-INVARIANT SCHS MODELS}

This section is devoted to a brief study of dualityinvariant models for $\mathcal{N}=2$ conformal superspin- $(s+1)$ gauge multiplets, with $s \geq 0$, in conformally flat backgrounds. ${ }^{8}$ Our approach will generalize the general theory of $\mathrm{U}(1)$ duality-invariant models for $\mathcal{N}=2$ supersymmetric nonlinear electrodynamics (superspin-0) developed in $[9,10,13,26]$. The essential information about $\mathcal{N}=2$ conformal superspace, which is used throughout this section, is collected in Appendix C.

We start with a review of SCHS gauge multiplets [52]. Given a non-negative integer $s$, the conformal superspin$(s+1)$ gauge multiplet is described by a real unconstrained prepotential $H_{\alpha(s) \dot{\alpha}(s)}$, which is a primary superfield, $K^{B} H_{\alpha(s) \dot{\alpha}(s)}=0$, defined modulo gauge transformations of the form

$s>0: \quad \delta_{\zeta} H_{\alpha(s) \dot{\alpha}(s)}=\nabla_{\left(\alpha_{1}\right.}^{i} \bar{\zeta}_{\left.\alpha_{2} \ldots \alpha_{s}\right) \dot{\alpha}(s) i}+\bar{\nabla}_{\left(\dot{\alpha}_{1}\right.}^{i} \zeta_{\left.\alpha(s) \dot{\alpha}_{2} \ldots \dot{\alpha}_{s}\right) i}$,

$s=0: \quad \delta_{\zeta} H=\nabla^{i j} \bar{\zeta}_{i j}+\bar{\nabla}^{i j} \zeta_{i j}$,

with the gauge parameters $\zeta_{\alpha(s) \dot{\alpha}(s-1) i}$ and $\zeta_{i j}$ being primary and complex unconstrained. Here we have defined the second-order operators

$$
\bar{\nabla}^{i j}:=\bar{\nabla}_{\dot{\alpha}}^{(i} \bar{\nabla}^{\dot{\alpha} j)}, \quad \nabla^{i j}:=\nabla^{\alpha(i} \bar{\nabla}_{\alpha}^{j)} .
$$

The gauge transformation laws (4.1) are superconformal provided

$$
\mathbb{D} H_{\alpha(s) \dot{\alpha}(s)}=-(s+2) H_{\alpha(s) \dot{\alpha}(s)} .
$$

Associated with the gauge prepotential $H_{\alpha(s) \dot{\alpha}(s)}$ is the chiral descendant [52]

$$
\begin{aligned}
\mathcal{W}_{\alpha(2 s+2)} & =\bar{\nabla}^{4} \nabla_{\left(\alpha_{1}\right.} \dot{\beta}_{1} \ldots \nabla_{\alpha_{s}} \dot{\beta}_{s} \nabla_{\alpha_{s+1} \alpha_{s+2}} H_{\left.\alpha_{s+3} \ldots \alpha_{2 s+2}\right) \dot{\beta}(s)}, \\
\bar{\nabla}_{j} \mathcal{W}_{\alpha(2 s+2)} & =0
\end{aligned}
$$

\footnotetext{
${ }^{8}$ See, e.g., [50,51] for the notions of $\mathcal{N}=2$ superspin and superisospin. For all superconformal multiplets considered in this section, their superisospin is equal to zero.
}

where we have introduced the chiral projection operator

$$
\bar{\nabla}^{4}:=\frac{1}{48} \bar{\nabla}^{i j} \bar{\nabla}_{i j}
$$

and the second-order operators

$$
\nabla_{\alpha \beta}:=\nabla_{(\alpha}^{k} \nabla_{\beta) k}, \quad \bar{\nabla}^{\dot{\alpha} \dot{\beta}}:=\bar{\nabla}_{k}^{(\dot{\alpha}} \bar{\nabla}^{\dot{\beta}) k} .
$$

It can be shown that $\mathcal{W}_{\alpha(2 s+2)}$ is primary in an arbitrary supergravity background,

$$
K^{B} \mathcal{W}_{\alpha(2 s+2)}=0, \quad \mathbb{D} \mathcal{W}_{\alpha(2 s+2)}=\mathcal{W}_{\alpha(2 s+2)} .
$$

However, the gauge transformations (4.1) leave $\mathcal{W}_{\alpha(2 s+2)}$ invariant, $\delta_{\zeta} \mathcal{W}_{\alpha(2 s+2)}=0$, only if the background curved superspace is conformally flat,

$$
W_{\alpha(2)}=0 \Rightarrow \delta_{\zeta} \mathcal{W}_{\alpha(2 s+2)}=0
$$

where $W_{\alpha(2)}$ is the background super-Weyl tensor, see Appendix C. Throughout this section, we will restrict ourselves to such a geometry.

We should point out that the gauge prepotential $H$ describes the linearized $\mathcal{N}=2$ conformal supergravity multiplet, and its field strength $\mathcal{W}_{\alpha(2)}$ is the linearized super-Weyl tensor. For $s>0$ we will refer to the fields strengths $\mathcal{W}_{\alpha(2 s+2)}$ as the linearized higher-spin superWeyl tensors.

The chiral field strength defined by (4.4) carries at least two spinor indices. A chiral scalar field strength $\mathcal{W}$ corresponds to the massless $\mathcal{N}=2$ vector multiplet [53]. It can be described in terms of the curved superspace analogue of Mezincescu's prepotential [54] (see also [55]), $V_{i j}=V_{j i}$, which is an unconstrained real $\mathrm{SU}(2)$ triplet, $\overline{V_{i j}}=V^{i j}=\varepsilon^{i k} \varepsilon^{j l} V_{k l}$. The expression for $\mathcal{W}$ in terms of $V_{i j}$ was found in [56] to be

$$
\mathcal{W}=\frac{1}{4} \bar{\nabla}^{4} \nabla^{i j} V_{i j}, \quad \bar{\nabla}_{j}^{\dot{\beta}} \mathcal{W}=0, \quad V_{i j}=V_{j i} .
$$

The field strength $\mathcal{W}$ defines a primary reduced chiral superfield of dimension +1 ,

$$
\nabla^{i j} \mathcal{W}=\bar{\nabla}^{i j} \overline{\mathcal{W}}, \quad \mathbb{D} \mathcal{W}=\mathcal{W}, \quad K_{A} \mathcal{W}=0,
$$

and is invariant under gauge transformations of the form [57]

$\delta_{\zeta} V^{i j}=\nabla^{\alpha}{ }_{k} \zeta_{\alpha}{ }^{k i j}+\bar{\nabla}_{\dot{\alpha} k} \bar{\zeta}^{\dot{\alpha} k i j}, \quad \zeta_{\alpha}{ }^{k i j}=\zeta_{\alpha}{ }^{(k i j)}$,

with $\zeta_{\alpha}{ }^{k i j}$ being primary and unconstrained modulo the algebraic condition given. In the flat-superspace limit, the gauge transformation law (4.11) reduces to that given in [54,55]. It is important to emphasize that $\mathcal{W}$ is invariant 
under the gauge transformations (4.11) in an arbitrary supergravity background.

The formalism we introduce below for nonlinear SCHS models generalizes the existing framework for $\mathrm{U}(1)$ duality-invariant theories of the vector multiplet. The latter have been studied extensively in $[9,10,26,28]$, thus we omit this special case from our considerations.

\section{A. U(1) duality-invariant models}

We consider a dynamical system describing the propagation of the superconformal gauge multiplet $H_{\alpha(s) \dot{\alpha}(s)}$, $s \geq 0$, in a conformally flat superspace. We require its action functional to take the form $S^{(s)}[\mathcal{W}, \overline{\mathcal{W}}]$, where the gauge-invariant chiral field strength $\mathcal{W}_{\alpha(2 s+2)}$ is given by Eq. (4.4).

It is important to note that the field strength (4.4) obeys the Bianchi identity

$$
\begin{aligned}
& \left.\nabla^{\beta_{1}} \dot{\alpha}_{1} \ldots \nabla^{\beta_{s}} \dot{\alpha}_{s}\right) \nabla^{\beta_{s+1} \beta_{s+2}} \mathcal{W}_{\alpha(s) \beta(s+2)} \\
& \quad=\nabla_{\left(\alpha_{1}\right.} \dot{\beta}_{1} \ldots \nabla_{\left.\alpha_{s}\right)} \dot{\beta}_{s} \bar{\nabla}^{\dot{\beta}_{s+1} \dot{\beta}_{s+2}} \overline{\mathcal{W}}_{\dot{\alpha}(s) \dot{\beta}(s+2)}
\end{aligned}
$$

We then assume that $S^{(s)}[\mathcal{W}, \mathcal{\mathcal { W }}]$ can be defined as a functional of a general chiral superfield $\mathcal{W}_{\alpha(2 s+2)}$ and its conjugate. This allows us to introduce the symmetric spinor

$$
\mathrm{i}_{\mathcal{M}_{\alpha(2 s+2)}}:=2 \frac{\delta S^{(s)}[\mathcal{W}, \overline{\mathcal{W}}]}{\delta \mathcal{W}^{\alpha(2 s+2)}}
$$

which defines an arbitrary variation of the action

$\delta S^{(s)}[\mathcal{W}, \overline{\mathcal{W}}]=\int \mathrm{d}^{4} x \mathrm{~d}^{2} \theta \mathcal{E} \delta \mathcal{W}^{\alpha(2 s+2)} \frac{\delta S^{(s)}[\mathcal{W}, \overline{\mathcal{W}}]}{\delta \mathcal{W}^{\alpha(2 s+2)}}+$ c.c.

where $\mathcal{E}$ is the $\mathcal{N}=2$ chiral integration measure. ${ }^{9}$ It follows that $\mathcal{M}_{\alpha(2 s+2)}$ is a primary chiral superfield,

$$
\begin{aligned}
K^{B} \mathcal{M}_{\alpha(2 s+2)} & =0, \quad \bar{\nabla}_{j}^{\dot{\beta}} \mathcal{M}_{\alpha(2 s+2)}=0, \\
\mathbb{D} \mathcal{M}_{\alpha(2 s+2)} & =\mathcal{M}_{\alpha(2 s+2)} .
\end{aligned}
$$

Varying the action with respect to the prepotential $H_{\alpha(s) \dot{\alpha}(s)}$ yields the equation of motion

$$
\begin{aligned}
& \left.\nabla^{\beta_{1}}{ }_{\left(\dot{\alpha}_{1}\right.} \ldots \nabla^{\beta_{s}} \dot{\alpha}_{s}\right) \nabla^{\beta_{s+1} \beta_{s+2}} \mathcal{M}_{\alpha(s) \beta(s+2)} \\
& \quad=\nabla_{\left(\alpha_{1}\right.} \dot{\beta}_{1} \ldots \nabla_{\left.\alpha_{s}\right)} \dot{\beta}_{s} \bar{\nabla}^{\dot{\beta}_{s+1} \dot{\beta}_{s+2}} \overline{\mathcal{M}}_{\dot{\alpha}(s) \dot{\beta}(s+2)} .
\end{aligned}
$$

This analysis above indicates that the functional form of (4.16) coincides with that of (4.12). As a result, the system

\footnotetext{
${ }^{9}$ The chiral and full superspace integrals are related to each according to Eq. (C9).
}

of Eqs. (4.12) and (4.16) is invariant under U(1) duality rotations:

$$
\delta_{\lambda} \mathcal{W}_{\alpha(2 s+2)}=\lambda \mathcal{M}_{\alpha(2 s+2)}, \delta_{\lambda} \mathcal{M}_{\alpha(2 s+2)}=-\lambda \mathcal{W}_{\alpha(2 s+2)},
$$

where $\lambda$ is a constant, real parameter. One may then obtain two equivalent expressions for the variation of $S^{(s)}[\mathcal{W}, \overline{\mathcal{W}}]$ with respect to (4.17)

$$
\begin{aligned}
\delta_{\lambda} S^{(s)}[\mathcal{W}, \overline{\mathcal{W}}] & =\frac{\mathrm{i} \lambda}{4} \int \mathrm{d}^{4} x \mathrm{~d}^{4} \theta \mathcal{E}\left\{\mathcal{W}^{2}-\mathcal{M}^{2}\right\}+\text { c.c. } \\
& =-\frac{\mathrm{i} \lambda}{2} \int \mathrm{d}^{4} x \mathrm{~d}^{4} \theta \mathcal{E} \mathcal{M}^{2}+\text { c.c. }
\end{aligned}
$$

as a generalization of similar derivations in nonlinear $\mathcal{N}=2$ supersymmetric electrodynamics $[9,10,13]$. This implies the self-duality equation

$$
\begin{aligned}
& \operatorname{Im} \int \mathrm{d}^{4} x \mathrm{~d}^{4} \theta \mathcal{E}\left\{\mathcal{W}^{\alpha(2 s+2)} \mathcal{W}_{\alpha(2 s+2)}\right. \\
& \left.+\mathcal{M}^{\alpha(2 s+2)} \mathcal{M}_{\alpha(2 s+2)}\right\}=0
\end{aligned}
$$

which must hold for a general chiral superfield $\mathcal{W}_{\alpha(2 s+2)}$ and its conjugate. Every solution $S^{(s)}[\mathcal{W}, \overline{\mathcal{W}}]$ of the selfduality equation describes a U(1) duality-invariant theory. Equation (4.19) allows one to prove, in complete analogy with the nonsupersymmetric analysis conducted in Sec. II B, that the U(1) duality-invariant theories are self-dual under Legendre transformations. The simplest solution of the self-duality equation (4.19) is the free $\mathcal{N}=2$ SCHS model (E8) proposed in [52], including the action for linearized conformal supergravity $(s=0)$.

\section{B. Auxiliary variable formulation}

This subsection is aimed at generalizing the auxiliary variable formalism employed in Sec. III B to construct selfdual $\mathcal{N}=1$ SCHS models to an $\mathcal{N}=2$ setting. ${ }^{10}$ To this end, we consider the action functional

$$
\begin{aligned}
S^{(s)}[\mathcal{W}, \overline{\mathcal{W}}, \boldsymbol{\eta}, \overline{\boldsymbol{\eta}}] & =(-1)^{s} \int \mathrm{d}^{4} x \mathrm{~d}^{4} \theta \mathcal{E}\left\{\boldsymbol{\eta} \mathcal{W}-\frac{1}{2} \boldsymbol{\eta}^{2}-\frac{1}{4} \mathcal{W}^{2}\right\} \\
& + \text { c.c. }+\mathcal{S}_{\text {int }}^{(s)}[\boldsymbol{\eta}, \overline{\boldsymbol{\eta}}]
\end{aligned}
$$

where we have introduced the auxiliary dimension-1 multiplet $\boldsymbol{\eta}_{\alpha(2 s+2)}$, which is primary and covariantly chiral,

\footnotetext{
${ }^{10}$ Such a setup was recently utilized in [28] to construct $\mathcal{N}=2$ superconformal duality-invariant models for an Abelian vector multiplet in a conformal supergravity background.
} 
$K^{B} \boldsymbol{\eta}_{\alpha(2 s+2)}=0, \quad \bar{\nabla}_{j}^{\dot{\beta}} \boldsymbol{\eta}_{\alpha(2 s+2)}=0, \quad \mathbb{D} \boldsymbol{\eta}_{\alpha(2 s+2)}=\boldsymbol{\eta}_{\alpha(2 s+2)}$.

By construction, the self-interaction $\mathcal{S}_{\text {int }}^{(s)}[\boldsymbol{\eta}, \overline{\boldsymbol{\eta}}]$ contains cubic and higher powers of $\boldsymbol{\eta}_{\alpha(2 s+2)}$ and its conjugate. Varying (4.20) with respect to this superfield yields

$$
\boldsymbol{\eta}_{\alpha(2 s+2)}=\mathcal{W}_{\alpha(2 s+2)}+(-1)^{s} \frac{\delta \mathcal{S}_{\text {int }}^{(s)}[\boldsymbol{\eta}, \overline{\boldsymbol{\eta}}]}{\delta \boldsymbol{\eta}^{\alpha(2 s+2)}} .
$$

Employing perturbation theory, Eq. (4.22) allows one to express $\boldsymbol{\eta}_{\alpha(2 s+2)}$ as a functional of $\boldsymbol{W}_{\alpha(2 s+1)}$ and its conjugate. This means that (4.20) is equivalent to a SCHS theory with action

$$
S^{(s)}[\mathcal{W}, \overline{\mathcal{W}}]=\frac{(-1)^{s}}{4} \int \mathrm{d}^{4} x \mathrm{~d}^{4} \theta \mathcal{E} \mathcal{W}^{2}+\text { c.c. }+S_{\text {int }}^{(s)}[\mathcal{W}, \overline{\mathcal{W}}]
$$

Thus, (4.20) and (4.23) provide two equivalent realizations of the same model.

We now analyze the self duality equation (4.19). It is easily verified that this constraint is equivalent to

$$
\operatorname{Im} \int \mathrm{d}^{4} x \mathrm{~d}^{4} \theta \mathcal{E} \boldsymbol{\eta}^{\alpha(2 s+2)} \frac{\delta \mathcal{S}_{\text {int }}^{(s)}[\boldsymbol{\eta}, \overline{\boldsymbol{\eta}}]}{\delta \boldsymbol{\eta}^{\alpha(2 s+2)}}=0 .
$$

Thus, the U(1) duality invariance of the model (4.20) is equivalent to the requirement that $\mathcal{S}_{\text {int }}^{(s)}[\boldsymbol{\eta}, \overline{\boldsymbol{\eta}}]$ is invariant under rigid $\mathrm{U}(1)$ phase transformations

$$
\mathcal{S}_{\text {int }}^{(s)}\left[\mathrm{e}^{\mathrm{i} \varphi} \boldsymbol{\eta}, \mathrm{e}^{-i \varphi} \bar{\eta}\right]=\mathcal{S}_{\text {int }}^{(s)}[\boldsymbol{\eta}, \overline{\boldsymbol{\eta}}], \quad \varphi \in \mathbb{R} .
$$

If we allow the action (4.20) to depend on a superconformal compensator, it is trivial to construct interactions satisfying the condition (4.25), for instance

$$
\begin{aligned}
\mathcal{S}_{\text {int }}^{(s)} & \left.\boldsymbol{\eta}, \overline{\boldsymbol{\eta}} ; \mathcal{W}_{0}, \overline{\mathcal{W}}_{0}\right] \\
& =\int \mathrm{d}^{4} x \mathrm{~d}^{4} \theta \mathrm{d}^{4} \bar{\theta} \boldsymbol{E} \mathfrak{F}\left(\frac{\boldsymbol{\eta}^{\alpha(2 s+2)} \boldsymbol{\eta}_{\alpha(2 s+2)} \overline{\boldsymbol{\eta}}_{\dot{\alpha}(2 s+2)} \overline{\boldsymbol{\eta}}^{\dot{\alpha}(2 s+2)}}{\left(\mathcal{W}_{0} \overline{\mathcal{W}}_{0}\right)^{2}}\right) .
\end{aligned}
$$

Here $\mathfrak{F}(x)$ is a real analytic function of a real variable, and $\mathcal{W}_{0}$ is the chiral field strength of a compensating vector multiplet.

It is not difficult to construct explicit examples of $\mathcal{N}=2$ superconformal and $\mathrm{U}(1)$ duality-invariant higher-spin theories, as a generalization of the $\mathcal{N}=2$ vector multiplet models presented in [28]. For simplicity, here we restrict our attention to $\mathcal{N}=2$ Minkowski superspace. Let us consider the self-interaction

$$
\mathcal{S}_{\text {int }}^{(s)}[\boldsymbol{\eta}, \overline{\boldsymbol{\eta}}]=c \int \mathrm{d}^{4} x \mathrm{~d}^{4} \theta \mathrm{d}^{4} \bar{\theta} \ln \boldsymbol{\eta}^{2} \ln \overline{\boldsymbol{\eta}}^{2},
$$

with $c$ a coupling constant. It is clearly invariant under rigid $\mathrm{U}(1)$ phase transformations, Eq. (4.25), and therefore it generates a $\mathrm{U}(1)$ duality-invariant model. It is also $\mathcal{N}=2$ superconformal, which may be checked using the properties of $\mathcal{N}=2$ superconformal transformations $[58,59]$. The above functional is well defined provided the auxiliary variables $\boldsymbol{\eta}_{\alpha(2 s+2)}$ are chosen to belong to the open domain $\boldsymbol{\eta}^{2}:=\boldsymbol{\eta}^{\alpha(2 s+2)} \boldsymbol{\eta}_{\alpha(2 s+2)} \neq 0$. A similar condition should be imposed in the case of nonsupersymmetric model (2.41).

\section{On $\mathcal{N}=2$ super ModMax theory}

So far, an $\mathcal{N}=2$ superconformal extension of the ModMax theory has not been constructed. Perhaps it does not exist (see the discussion in [28]), the main reason being the fact that the full $\mathcal{N}=2$ superspace measure is dimensionless. However, if the action functional is allowed to depend on compensators, an $\mathcal{N}=2$ supersymmetric extension of the ModMax theory can be introduced. Specifically, let us consider the following $\mathcal{N}=2$ vector multiplet model $^{11}$ :

$$
\begin{aligned}
& S\left[\mathcal{W}, \overline{\mathcal{W}}, \boldsymbol{\eta}, \overline{\boldsymbol{\eta}} ; \mathcal{W}_{0}, \overline{\mathcal{W}}_{0}\right] \\
& =\frac{1}{2} \int \mathrm{d}^{4} x \mathrm{~d}^{4} \theta \mathcal{E}\left\{\boldsymbol{\eta} \mathcal{W}-\frac{1}{2} \boldsymbol{\eta}^{2}-\frac{1}{4} \mathcal{W}^{2}\right\}+\text { c.c. } \\
& \quad+\mathcal{S}_{\text {int }}\left[\boldsymbol{\eta}, \overline{\boldsymbol{\eta}} ; \mathcal{W}_{0}, \overline{\mathcal{W}}_{0}\right] \\
& \mathcal{S}_{\text {int }}\left[\boldsymbol{\eta}, \overline{\boldsymbol{\eta}} ; \mathcal{W}_{0}, \overline{\mathcal{W}}_{0}\right] \\
& \quad=\kappa \int \mathrm{d}^{4} x \mathrm{~d}^{4} \theta \mathrm{d}^{4} \bar{\theta} \boldsymbol{E} \frac{\boldsymbol{\eta}^{2} \overline{\boldsymbol{\eta}}^{2}}{\mathcal{W}_{0} \overline{\mathcal{W}}_{0} \sqrt{\nabla^{4}\left[\frac{\boldsymbol{\eta}}{\mathcal{W}_{0}}\right]^{2} \bar{\nabla}^{4}\left[\frac{\bar{\eta}}{\mathcal{W}_{0}}\right]^{2}}},
\end{aligned}
$$

where $\kappa \in \mathbb{R}$ is the coupling constant. We are going to show that this model incorporates $\mathcal{N}=1$ superconformal ModMax theory, Eq. (3.27), in the sense described below.

The field strength $\mathcal{W}$ of the $\mathcal{N}=2$ vector multiplet contains two independent $\mathcal{N}=1$ chiral components, $\Phi$ and $\mathcal{W}_{\alpha}$, defined by

$$
\begin{aligned}
\left.\mathcal{W}\right|_{\theta_{\underline{2}}=0} & =\sqrt{2} \Phi \\
\left.\nabla \frac{2}{\alpha} \mathcal{W}\right|_{\theta_{\underline{2}}=0} & =\left.2 \mathrm{i} \mathcal{W}_{\alpha} \Rightarrow\left(\nabla^{2}\right)^{2} \mathcal{W}\right|_{\theta_{\underline{2}}=0}=\sqrt{2} \bar{\nabla}^{2} \bar{\Phi}
\end{aligned}
$$

Here $\mathcal{W}_{\alpha}$ is the chiral field strength of the $\mathcal{N}=1$ vector multiplet, $\nabla^{\alpha} \mathcal{W}_{\alpha}=\bar{\nabla}_{\dot{\alpha}} \overline{\mathcal{W}}^{\dot{\alpha}}$. The auxiliary $\mathcal{N}=2$ chiral superfield $\boldsymbol{\eta}$ contains three independent $\mathcal{N}=1$ chiral components, $\chi, \eta_{\alpha}$ and $\Psi$, defined by

\footnotetext{
${ }^{11}$ Our normalization of the quadratic part of the vector multiplet action follows [28].
} 
$\left.\boldsymbol{\eta}\right|_{\theta_{\underline{2}}=0}=\sqrt{2} \chi,\left.\quad \nabla_{\alpha}^{2} \boldsymbol{\eta}\right|_{\theta_{\underline{2}}=0}=2 \mathrm{i} \eta_{\alpha}, \quad-\left.\frac{1}{4}\left(\nabla^{2}\right)^{2} \boldsymbol{\eta}\right|_{\theta_{\underline{2}}=0}=\Psi$.

Using the standard $\mathcal{N}=2 \rightarrow \mathcal{N}=1$ reduction rules

$$
\begin{aligned}
\int \mathrm{d}^{4} x \mathrm{~d}^{4} \theta \mathcal{E} \mathcal{L}_{c} & =-\left.\frac{1}{4} \int \mathrm{d}^{4} x \mathrm{~d}^{2} \theta \mathcal{E}\left(\nabla^{2}\right)^{2} \mathcal{L}_{c}\right|_{\theta_{\underline{2}}=0}, \\
\bar{\nabla}_{i}^{\dot{\alpha}} \mathcal{L}_{c} & =0 \\
\int \mathrm{d}^{4} x \mathrm{~d}^{4} \theta \mathrm{d}^{4} \bar{\theta} \mathcal{E} \mathcal{L} & =\left.\frac{1}{16} \int \mathrm{d}^{4} x \mathrm{~d}^{2} \theta \mathrm{d}^{2} \bar{\theta} E\left(\nabla^{2}\right)^{2}\left(\bar{\nabla}_{\underline{2}}\right)^{2} \mathcal{L}\right|_{\theta_{\underline{2}}=0},
\end{aligned}
$$

we may reduce the action (4.28) to $\mathcal{N}=1$ superspace. Here we restrict our attention to the $\mathcal{N}=1$ vector multiplet and switch off the $\mathcal{N}=1$ scalar multiplet, $\Phi=0$. Then it may be shown that setting $\chi=0$ and $\Psi=0$ gives a solution of the equations of motion for these auxiliary superfields. ${ }^{12}$ The resulting $\mathcal{N}=1$ superspace action is

$$
\begin{aligned}
S[\mathcal{W}, \overline{\mathcal{W}}, \eta, \bar{\eta}]= & \int \mathrm{d}^{4} x \mathrm{~d}^{2} \theta \mathcal{E}\left\{\eta \mathcal{W}-\frac{1}{2} \eta^{2}-\frac{1}{4} \mathcal{W}^{2}\right\}+\text { c.c. } \\
& +\kappa \int \mathrm{d}^{4} x \mathrm{~d}^{2} \theta \mathrm{d}^{2} \bar{\theta} E \frac{\eta^{2} \bar{\eta}^{2}}{\Upsilon^{2} \sqrt{v \bar{v}}}
\end{aligned}
$$

Here $v$ is defined as in (3.24b), and we utilize the realization $\Upsilon=S_{0} \bar{S}_{0}$ for the compensator, with $\overline{\mathcal{W}}_{0} \mid=S_{0}$. The action (4.32) was proposed in [28] to describe $\mathcal{N}=1$ superconformal U(1) duality-invariant electrodynamics. Upon eliminating the auxiliary chiral spinor $\eta_{\alpha}$ and its conjugate, this model was shown in [28] to reduce to $\mathcal{N}=1$ superconformal ModMax theory, Eq. (3.27), introduced in [31].

\section{CONCLUSION}

In this paper we have generalized the general theory of U(1) duality-invariant nonlinear electrodynamics [3-8] and its $\mathcal{N}=1$ and $\mathcal{N}=2$ supersymmetric extensions [9-13,26,27] to the cases of bosonic conformal spin-s gauge fields, $s \geq 2$, and their $\mathcal{N}=1$ and $\mathcal{N}=2$ superconformal cousins. These self-dual higher-spin theories share several important features of duality-invariant electrodynamics, such as self-duality under Legendre transformations. The crucial difference between the U(1) duality-invariant models for electrodynamics and their higher-spin counterparts is that the former are consistently defined on arbitrary gravitational backgrounds, while the latter are formulated in a conformally flat space.

In the nonsupersymmetric case, we presented several families of self-dual models. The simplest of these is (2.37),

\footnotetext{
${ }^{12}$ This follows from the fact that the $\mathcal{N}=1$ superspace reduction of (4.28) does not have terms linear in $\chi, \Psi$ and their conjugates provided $\Phi=0$.
}

which is a higher-spin generalization of ModMax electrodynamics [29]. We also introduced a two-parameter family of models for the conformal graviton (2.40) and provided insights on the construction of new families of multiparameter duality invariant models for conformal higher-spin fields. Finally, in the supersymmetric case, we generalized the construction of $\mathcal{N}=1$ superconformal duality invariant electrodynamics [28,31] to higher spins (3.28). A distinguishing feature of this family of models is that, at the component level, their purely bosonic sectors vanish, which may be verified via a component reduction.

It should be emphasized that the literature on duality is huge and, unfortunately, it is not possible to mention all publications here. In this paper we were interested in duality as a continuous symmetry of the equations of motion. There exists a different approach to duality as a manifest symmetry of the action. The latter was advocated in [60-66]. Another important approach was inspired by early examples of dual field theories [67-73] and resulted in various dual formulations for massless, massive and partially massless higherspin field theories, see [74-80] and references therein. This approach corresponds to discrete dualities.

There may be several generalizations of our results that are modeled on similar properties of duality-invariant theories of spin-1 gauge fields, see [81] for a review. In particular, we recall that, given a U(1) duality-invariant model for nonlinear electrodynamics, its compact duality group $\mathrm{U}(1)$ can be enhanced to the noncompact $\operatorname{SL}(2, \mathbb{R})$ group by coupling the electromagnetic field to the dilaton and axion [3-5]. It may be shown that this construction naturally extends to the higher-spin case. We should point out that a generalization of the method of [6-8] to the case of $\operatorname{SL}(2, \mathbb{R})$ duality was given by Ivanov, Lechtenfeld and Zupnik [82].

Fundamental to our analysis in this paper has been the formalism of conformal (super) space, which trivializes calculations in backgrounds with vanishing (super) Weyl tensor. For the purpose of applications, however, it is often useful to work with Lorentz covariant derivatives as opposed to their conformally covariant counterparts. The process of translating results expressed in terms of the latter to the former is known as degauging. The general prescription for such an analysis is well known (see e.g. $[35,83,84]$ ), however such calculations are often highly nontrivial on generic curved backgrounds. If the background geometry is restricted by turning off several components of the curvature, the resulting computations are greatly simplified. To this end, we now provide a dictionary to translate our main results to the AdS (super)space. ${ }^{13}$

We begin with the nonsupersymmetric story. The geometry of $\mathrm{AdS}_{4}$ is encoded within the Lorentz covariant derivatives $\mathcal{D}_{a}$, which obey the algebra

\footnotetext{
${ }^{13}$ The conformal flatness of $\mathcal{N}$-extended AdS superspace in four dimensions was established in [85] and further elaborated in [86].
} 


$$
\left[\mathcal{D}_{\alpha \dot{\alpha}}, \mathcal{D}_{\beta \dot{\beta}}\right]=-2 \mu \bar{\mu}\left(\varepsilon_{\dot{\alpha} \dot{\beta}} M_{\alpha \beta}+\varepsilon_{\alpha \beta} \bar{M}_{\dot{\alpha} \dot{\beta}}\right),
$$

where $\mu$ is a nonzero complex constant with the dimension of mass encoding the curvature of the spacetime and $\mu \bar{\mu}$ is proportional to the scalar curvature. It may be shown that in this geometry, Eq. (2.1) takes the form

$$
\mathcal{C}_{\alpha(2 s)}=\mathcal{D}_{\left(\alpha_{1}\right.} \dot{\beta}_{1} \ldots \mathcal{D}_{\alpha_{s}} \dot{\beta}_{s} h_{\left.\alpha_{s+1} \ldots \alpha_{2 s}\right) \dot{\beta}(s)} .
$$

Further, $\mathcal{C}_{\alpha(2 s)}$ satisfies the Bianchi identity (2.6)

$$
\left.\mathcal{D}^{\beta_{1}}{\left(\dot{\alpha}_{1}\right.}_{1} \ldots \mathcal{D}^{\beta_{s}} \dot{\alpha}_{s}\right) \mathcal{C}_{\alpha(s) \beta(s)}=\mathcal{D}_{\left(\alpha_{1}\right.} \dot{\beta}_{1} \ldots \mathcal{D}_{\left.\alpha_{s}\right)} \dot{\beta}_{s} \overline{\mathcal{C}}_{\dot{\alpha}(s) \dot{\beta}(s)} .
$$

Next, the geometry of the $\mathcal{N}=1$ AdS superspace, $\operatorname{AdS}^{4 \mid 4}$, is described by the covariant derivatives $\mathcal{D}_{A}=$ $\left(\mathcal{D}_{a}, \mathcal{D}_{\alpha}, \overline{\mathcal{D}}^{\dot{\alpha}}\right)$, which obey the algebra (see, e.g., [87])

$$
\begin{aligned}
& \left\{\mathcal{D}_{\alpha}, \mathcal{D}_{\beta}\right\}=-4 \bar{\mu} M_{\alpha \beta}, \quad\left\{\overline{\mathcal{D}}_{\dot{\alpha}}, \overline{\mathcal{D}}_{\dot{\beta}}\right\}=4 \mu \bar{M}_{\dot{\alpha} \dot{\beta}}, \\
& \left\{\mathcal{D}_{\alpha}, \overline{\mathcal{D}}_{\dot{\alpha}}\right\}=2 \mathrm{i} \mathcal{D}_{\alpha \dot{\alpha}}, \\
& {\left[\mathcal{D}_{\alpha}, \mathcal{D}_{\beta \dot{\beta}}\right]=\mathrm{i} \bar{\mu} \varepsilon_{\alpha \beta} \overline{\mathcal{D}}_{\dot{\beta}}, \quad\left[\overline{\mathcal{D}}_{\dot{\alpha}}, \overline{\mathcal{D}}_{\beta \dot{\beta}}\right]=-\mathrm{i} \mu \varepsilon_{\dot{\alpha} \dot{\beta}} \mathcal{D}_{\beta},}
\end{aligned}
$$

where $\mu$ is a nonzero constant having the dimension of mass. In this supergeometry the field strengths $\mathcal{W}_{\alpha(2 s+1)}$ take the form [88]

$$
\mathcal{W}_{\alpha(2 s+1)}=-\frac{1}{4}\left(\overline{\mathcal{D}}^{2}-4 \mu\right) \mathcal{D}_{\left(\alpha_{1}\right.} \dot{\beta}_{1} \ldots \mathcal{D}_{\alpha_{s}} \dot{\beta}_{s} \mathcal{D}_{\alpha_{s+1}} H_{\left.\alpha_{s+2} \ldots \alpha_{2 s+1}\right) \dot{\beta}(s)},
$$

and obey the Bianchi identity

$$
\begin{aligned}
& \left.\mathrm{i} \mathcal{D}^{\beta_{1}} \dot{\alpha}_{1} \ldots \mathcal{D}^{\beta_{s}} \dot{\alpha}_{s}\right) \mathcal{D}^{\beta_{s+1}} \mathcal{W}_{\alpha(s) \beta(s+1)} \\
& \quad=-\mathrm{i} \mathcal{D}_{\left(\alpha_{1}\right.} \dot{\beta}_{1} \ldots \mathcal{D}_{\left.\alpha_{s}\right)} \dot{\beta}_{s} \overline{\mathcal{D}}^{\dot{\beta}_{s+1}} \overline{\mathcal{W}}_{\dot{\alpha}(s) \dot{\beta}(s+1)} .
\end{aligned}
$$

Finally, we consider $\operatorname{Ad} S^{4 \mid 8}$, the $\mathcal{N}=2$ AdS superspace. Recall that its geometry is encoded within the covariant derivatives $\mathcal{D}_{A}=\left(\mathcal{D}_{a}, \mathcal{D}_{\alpha}^{i}, \overline{\mathcal{D}}_{i}^{\dot{\alpha}}\right)$, which obey the algebra (see [86] for the technical details):

$$
\begin{aligned}
& \left\{\mathcal{D}_{\alpha}^{i}, \mathcal{D}_{\beta}^{j}\right\}=4 S^{i j} M_{\alpha \beta}+2 \varepsilon_{\alpha \beta} \varepsilon^{i j} S^{k l} J_{k l}, \\
& \left\{\mathcal{D}_{\alpha}^{i}, \overline{\mathcal{D}}_{j}^{\dot{\beta}}\right\}=-2 \mathrm{i} \delta_{j}^{i} \mathcal{D}_{\alpha}^{\dot{\beta}},
\end{aligned}
$$

where $S^{i j}$ is a nonzero covariantly constant isotriplet, $S^{j i}=S^{i j}$, satisfying the integrability condition $\left[S, S^{\dagger}\right]=0$, with $S=\left(S^{i}{ }_{j}\right) .{ }^{14}$ Here, the field strengths $\mathcal{W}_{\alpha(2 s+2)}$ take the form

\footnotetext{
${ }^{14}$ The integrability condition implies that $S^{i j}$ can be chosen to be real, $\overline{S^{i j}}=S_{i j}=\varepsilon_{i k} \varepsilon_{j l} S^{k l}$. However we will not impose the reality condition.
}

$$
\begin{aligned}
\mathcal{W}_{\alpha(2 s+2)}= & \frac{1}{48}\left(\overline{\mathcal{D}}^{i j}+4 \bar{S}^{i j}\right) \overline{\mathcal{D}}_{i j} \\
& \times \mathcal{D}_{\left(\alpha_{1}\right.} \dot{\beta}_{1} \ldots \mathcal{D}_{\alpha_{s}} \dot{\beta}_{s} \mathcal{D}_{\alpha_{s+1} \alpha_{s+2}} H_{\left.\alpha_{s+3} \ldots \alpha_{2 s+2}\right) \dot{\beta}(s)},
\end{aligned}
$$

and satisfy the Bianchi identity

$$
\begin{aligned}
& \left.\mathcal{D}^{\beta_{1}}{ }_{\left(\dot{\alpha}_{1}\right.} \ldots \mathcal{D}^{\beta_{s}} \dot{\alpha}_{s}\right) \mathcal{D}^{\beta_{s+1} \beta_{s+2}} \mathcal{W}_{\alpha(s) \beta(s+2)} \\
& \quad=\mathcal{D}_{\left(\alpha_{1}\right.} \dot{\beta}_{1} \ldots \mathcal{D}_{\left.\alpha_{s}\right)} \dot{\beta}_{s} \overline{\mathcal{D}}^{\dot{\beta}_{s+1}} \dot{\beta}_{s+2} \overline{\mathcal{W}}_{\dot{\alpha}(s) \dot{\beta}(s+2)}
\end{aligned}
$$

Throughout this work we have restricted our attention to the analysis of models described by real gauge prepotentials. However, our construction readily extends itself to the complex case. In particular, one may consider a complex CHS gauge prepotential $\phi_{\alpha(m) \dot{\alpha}(n)}$, with $m, n \geq 1$ and $m \neq n$ $[35,43,89]$. It is a primary field, $K_{b} \phi_{\alpha(m) \dot{\alpha}(n)}=0$, defined modulo

$$
\delta_{\ell} \phi_{\alpha(m) \dot{\alpha}(n)}=\nabla_{\left(\alpha _ { 1 } \left(\dot{\alpha}_{1}\right.\right.} \ell_{\left.\left.\alpha_{2} \ldots \alpha_{m}\right) \dot{\alpha}_{2} \ldots \dot{\alpha}_{n}\right)},
$$

where the gauge parameter $\ell_{\alpha(m-1) \dot{\alpha}(n-1)}$ is also primary. Conformal invariance of (5.10) uniquely fixes the dimension of the gauge field, $\mathbb{D} \phi_{\alpha(m) \dot{\alpha}(n)}=$ $\left(2-\frac{1}{2}(m+n)\right) \phi_{\alpha(m) \dot{\alpha}(n)}$. The action functional describing its dynamics is required to take the form $S^{(m, n)}[\hat{\mathcal{C}}, \check{\mathcal{C}}, \overline{\mathcal{C}}, \overline{\mathcal{C}}]$, where we have made the definitions

$$
\begin{aligned}
& \hat{\mathcal{C}}_{\alpha(m+n)}=\nabla_{\left(\alpha_{1}\right.} \dot{\beta}_{1} \ldots \nabla_{\alpha_{n}} \dot{\beta}_{n} \phi_{\left.\alpha_{n+1} \ldots \alpha_{m+n}\right) \dot{\beta}(n)}, \\
& \check{\mathcal{C}}_{\alpha(m+n)}=\nabla_{\left(\alpha_{1}\right.} \dot{\beta}_{1} \ldots \nabla_{\alpha_{m}} \dot{\beta}_{m} \bar{\phi}_{\left.\alpha_{m+1} \ldots \alpha_{m+n}\right) \dot{\beta}(m)} .
\end{aligned}
$$

The descendants introduced above are primary in generic backgrounds,

$$
K_{b} \hat{\mathcal{C}}_{\alpha(m+n)}=0, \quad \mathbb{D} \hat{\mathcal{C}}_{\alpha(m+n)}=\left(2+\frac{1}{2}(n-m)\right) \hat{\mathcal{C}}_{\alpha(m+n)}
$$

$K_{b} \breve{\mathcal{C}}_{\alpha(m+n)}=0, \quad \mathbb{\mathbb { D }} \check{\mathcal{C}}_{\alpha(m+n)}=\left(2+\frac{1}{2}(m-n)\right) \check{\mathcal{C}}_{\alpha(m+n)}$,

and gauge-invariant in all conformally flat ones,

$$
C_{\alpha(4)}=0 \Rightarrow \delta_{\ell} \hat{\mathcal{C}}_{\alpha(m+n)}=\delta_{\ell} \check{\mathcal{C}}_{\alpha(m+n)}=0 .
$$

It is important to note that the field strengths (5.11) also obey the Bianchi identity

$\left.\nabla^{\beta_{1}} \dot{\alpha}_{1} \ldots \nabla^{\beta_{m}} \dot{\alpha}_{m}\right) \hat{\mathcal{C}}_{\alpha(n) \beta(m)}=\nabla_{\left(\alpha_{1}\right.} \dot{\beta}_{1} \ldots \nabla_{\left.\alpha_{n}\right)} \dot{\beta}_{n} \overline{\mathcal{C}}_{\dot{\alpha}(m) \dot{\beta}(n)}$. 
Now, considering $S^{(m, n)}[\hat{\mathcal{C}}, \check{\mathcal{C}}, \overline{\hat{\mathcal{C}}}, \overline{\mathcal{C}}]$ as a functional of the unconstrained fields $\hat{\mathcal{C}}_{\alpha(m+n)}, \breve{\mathcal{C}}_{\alpha(m+n)}$ and their conjugates, we may introduce the primary fields

$$
\begin{aligned}
\mathrm{i}^{m+n+1} \hat{\mathcal{M}}_{\alpha(m+n)} & :=\frac{\delta S^{(m, n)}[\hat{\mathcal{C}}, \check{\mathcal{C}}, \overline{\hat{\mathcal{C}}}, \overline{\mathcal{C}}]}{\delta \check{\mathcal{C}}^{\alpha(m+n)}}, \\
\mathrm{i}^{m+n+1} \check{\mathcal{M}}_{\alpha(m+n)} & :=\frac{\delta S^{(m, n)}[\hat{\mathcal{C}}, \check{\mathcal{C}}, \overline{\hat{\mathcal{C}}}, \overline{\mathcal{C}}]}{\delta \hat{\mathcal{C}}^{\alpha(m+n)}},
\end{aligned}
$$

where we have made the definition

$$
\begin{aligned}
\delta S^{(m, n)}[\hat{\mathcal{C}}, \check{\mathcal{C}}, \overline{\hat{\mathcal{C}}}, \overline{\mathcal{C}}]= & \int \mathrm{d}^{4} x e\left\{\delta \hat{\mathcal{C}}^{\alpha(m+n)} \frac{\delta S^{(m, n)}[\hat{\mathcal{C}}, \check{\mathcal{C}}, \overline{\hat{\mathcal{C}}}, \overline{\mathcal{C}}]}{\delta \hat{\mathcal{C}}^{\alpha(m+n)}}\right. \\
& \left.+\delta \check{\mathcal{C}}^{\alpha(m+n)} \frac{\delta S^{(m, n)}[\hat{\mathcal{C}}, \check{\mathcal{C}}, \overline{\hat{\mathcal{C}}}, \overline{\mathcal{C}}]}{\delta \check{\mathcal{C}}(m+n)}\right\}+ \text { c.c. }
\end{aligned}
$$

The conformal properties of the fields (5.15) are

$$
\begin{aligned}
K_{b} \hat{\mathcal{M}}_{\alpha(m+n)} & =0 \\
\mathbb{D} \hat{\mathcal{M}}_{\alpha(m+n)} & =\left(2+\frac{1}{2}(n-m)\right) \hat{\mathcal{M}}_{\alpha(m+n)} ; \\
K_{b} \check{\mathcal{M}}_{\alpha(m+n)} & =0 \\
\mathbb{D} \check{\mathcal{M}}_{\alpha(m+n)} & =\left(2+\frac{1}{2}(m-n)\right) \check{\mathcal{M}}_{\alpha(m+n)} .
\end{aligned}
$$

Varying $S^{(m, n)}[\hat{\mathcal{C}}, \check{\mathcal{C}}, \overline{\hat{\mathcal{C}}}, \overline{\mathcal{C}}]$ with respect to $\phi_{\alpha(m) \dot{\alpha}(n)}$ yields

$$
\nabla_{\left(\dot{\alpha}_{1} \ldots \nabla^{\beta_{m}} \dot{\alpha}_{m}\right)}^{\beta_{1}} \hat{\mathcal{M}}_{\alpha(n) \beta(m)}=\nabla_{\left(\alpha_{1}\right.} \dot{\beta}_{1} \ldots \nabla_{\left.\alpha_{n}\right)} \dot{\beta}_{n} \overline{\mathcal{M}}_{\dot{\alpha}(m) \dot{\beta}(n)} .
$$

It is clear from the discussion above that the system of Eqs. (5.14) and (5.18) is invariant under the U(1) duality rotations

$$
\begin{aligned}
& \delta_{\lambda} \hat{\mathcal{C}}_{\alpha(m+n)}=\lambda \hat{\mathcal{M}}_{\alpha(m+n)}, \quad \delta_{\lambda} \check{\mathcal{C}}_{\alpha(m+n)}=\lambda \check{\mathcal{M}}_{\alpha(m+n)}, \\
& \delta_{\lambda} \hat{\mathcal{M}}_{\alpha(m+n)}=-\lambda \hat{\mathcal{C}}_{\alpha(m+n)}, \quad \delta_{\lambda} \check{\mathcal{M}}_{\alpha(m+n)}=-\lambda \check{\mathcal{C}}_{\alpha(m+n)} .
\end{aligned}
$$

One may then perform similar analyses to those undertaken in Sec. II and construct U(1) duality-invariant nonlinear models for such fields. ${ }^{15}$ They satisfy the self-duality equation

\footnotetext{
${ }^{15}$ We mention in passing that such a construction can also be uplifted to the case of a SCHS theory described by a complex prepotential, such as those of $[35,48,90]$.
}

$$
\begin{aligned}
& \mathrm{i}^{m+n+1} \int \mathrm{d}^{4} x e\left\{\hat{\mathcal{C}}^{\alpha(m+n)} \breve{\mathcal{C}}_{\alpha(m+n)}+\hat{\mathcal{M}}^{\alpha(m+n)} \check{\mathcal{M}}_{\alpha(m+n)}\right\} \\
& \quad+\text { c.c. }=0
\end{aligned}
$$

which must hold for unconstrained fields $\hat{\mathcal{C}}_{\alpha(m+n)}$ and $\check{\mathcal{C}}_{\alpha(m+n)}$. The simplest solution of this equation is the free CHS action

$S^{(m, n)}[\hat{\mathcal{C}}, \check{\mathcal{C}}, \overline{\hat{\mathcal{C}}}, \overline{\mathcal{C}}]=\mathrm{i}^{m+n} \int \mathrm{d}^{4} x e \hat{\mathcal{C}}^{\alpha(m+n)} \check{\mathcal{C}}_{\alpha(m+n)}+$ c.c.,

which reduces to (E1) for $m=n=s$ and real prepotential.

$\mathrm{U}(1)$ duality-invariant actions of the type considered above naturally arise at the component level of supersymmetric self-dual theories, such as those discussed in Secs. III and IV. Such considerations are beyond the scope of the current paper and present an interesting avenue for further work. It would also be interesting to study duality-invariant models of the higher-depth (super)fields of $[35,91]$.

\section{ACKNOWLEDGMENTS}

We thank the referee for useful comments and suggestions. S. K. is grateful to Stefan Theisen and Arkady Tseytlin for discussions. His work is supported in part by the Australian Research Council, Project No. DP200101944. The work of E. R. is supported by the Hackett Postgraduate Scholarship UWA, under the Australian Government Research Training Program.

\section{APPENDIX A: CONFORMAL SPACE}

This appendix reviews the salient details of conformal geometry in four dimensions [92] pertinent to this work. ${ }^{16}$ We adopt the spinor conventions of [87], which are similar to those of [94]. We consider a curved spacetime $\mathcal{M}^{4}$ parametrized by local coordinates $x^{m}$. The structure group is chosen to be $\mathrm{SU}(2,2)$, whose Lie algebra is spanned by the translation $P_{a}$, Lorentz $M_{a b}$, dilatation $\mathbb{D}$ and the special conformal $K_{a}$ generators. The covariant derivatives $\nabla_{a}$ then take the form

$$
\nabla_{a}=e_{a}{ }^{m} \partial_{m}-\frac{1}{2} \omega_{a}{ }^{b c} M_{b c}-\mathfrak{b}_{a} \mathbb{D}-\mathfrak{f}_{a}{ }^{b} K_{b}
$$

where $e_{a}{ }^{m}$ is the inverse vielbein, $\omega_{a}{ }^{b c}$ the Lorentz spin connection, $\mathfrak{b}_{a}$ the dilatation connection and $\mathfrak{f}_{a}{ }^{b}$ the special conformal connection. The commutation relations of $\nabla_{a}$

\footnotetext{
${ }^{16}$ See also [32] for a pedagogical review of conformal (super)gravity and $[35,83,93]$ for the modern formulation of conformal geometry we use.
} 
with the generators $M_{b c}, \mathbb{D}$ and $K_{b}$ are obtained from those of $P_{a}$ with the same generators by replacing $P_{a} \rightarrow \nabla_{a}$.

The covariant derivatives (A1) obey the commutation relations

$$
\begin{aligned}
{\left[\nabla_{\alpha \dot{\alpha}}, \nabla_{\beta \dot{\beta}}\right]=} & -\left(\varepsilon_{\dot{\alpha} \dot{\beta}} C_{\alpha \beta \gamma \delta} M^{\gamma \delta}+\varepsilon_{\alpha \beta} \bar{C}_{\dot{\alpha} \dot{\beta} \dot{\gamma} \dot{\delta}} \bar{M}^{\dot{\gamma} \dot{\delta}}\right) \\
& -\frac{1}{4}\left(\varepsilon_{\dot{\alpha} \dot{\beta}} \nabla^{\delta \dot{\gamma}} C_{\alpha \beta \delta} \gamma+\varepsilon_{\alpha \beta} \nabla^{\gamma \dot{\delta}} \bar{C}_{\dot{\alpha} \dot{\beta} \dot{\delta}} \dot{\gamma}\right) K_{\gamma \dot{\gamma}}
\end{aligned}
$$

Here $C_{\alpha \beta \gamma \delta}$ and $\bar{C}_{\dot{\alpha} \dot{\beta} \dot{\gamma} \dot{\delta}}$ are the self-dual and anti-self-dual parts of the Weyl tensor $C_{a b c d}$, and are primary. We remind the reader that a field $\varphi$ (with suppressed indices) is said to be primary if it obeys

$$
K_{\alpha \dot{\alpha}} \varphi=0 .
$$

The commutation relations (A2) should be accompanied by the relations

$$
\begin{aligned}
{\left[\mathbb{D}, \nabla_{\alpha \dot{\alpha}}\right] } & =\nabla_{\alpha \dot{\alpha}}, \\
{\left[K_{\alpha \dot{\alpha}}, \nabla_{\beta \dot{\beta}}\right] } & =4\left(\varepsilon_{\dot{\alpha} \dot{\beta}} M_{\alpha \beta}+\varepsilon_{\alpha \beta} \bar{M}_{\dot{\alpha} \dot{\beta}}-\varepsilon_{\alpha \beta} \varepsilon_{\dot{\alpha} \dot{\beta}} \mathbb{D}\right),
\end{aligned}
$$

and we recall that the Lorentz generators act on vectors and Weyl spinors as follows:

$$
M_{a b} V_{c}=2 \eta_{c[a} V_{b]}, \quad M_{\alpha \beta} \psi_{\gamma}=\varepsilon_{\gamma(\alpha} \psi_{\beta)}, \quad \bar{M}_{\dot{\alpha} \dot{\beta}} \bar{\psi}_{\dot{\gamma}}=\varepsilon_{\dot{\gamma}(\dot{\alpha}} \bar{\psi}_{\dot{\beta})} .
$$

\section{APPENDIX B: $\mathcal{N}=1$ CONFORMAL SUPERSPACE}

In this appendix we review the elements of the $\mathcal{N}=1$ conformal superspace approach to off-shell conformal supergravity relevant to this work. For more details we refer the reader to the original paper [83] (see also Appendix A of [48]).

Consider a curved $\mathcal{N}=1$ superspace $\mathcal{M}^{4 \mid 4}$ parametrized by local coordinates $z^{M}=\left(x^{m}, \theta^{\mu}, \bar{\theta}_{\dot{\mu}}\right)$. The structure group is chosen to be $\mathrm{SU}(2,2 \mid 1)$. Its corresponding superalgebra is spanned by the translation $P_{A}=\left(P_{a}, Q_{\alpha}, \bar{Q}^{\dot{\alpha}}\right)$, Lorentz $M_{a b}$, dilatation $\mathbb{D}$, R-symmetry $Y$ and the special conformal $K^{A}=\left(K^{a}, S^{\alpha}, \bar{S}_{\dot{\alpha}}\right)$ generators. The covariant derivatives $\nabla_{A}$ then have the form

$$
\begin{aligned}
\nabla_{A} & =\left(\nabla_{a}, \nabla_{\alpha}, \bar{\nabla}^{\dot{\alpha}}\right) \\
& =E_{A}{ }^{M} \partial_{M}-\frac{1}{2} \Omega_{A}{ }^{b c} M_{b c}-\mathrm{i} \Phi_{A} Y-B_{A} \mathbb{D}-\mathfrak{\mho}_{A B} K^{B},
\end{aligned}
$$

where $E_{A}{ }^{M}$ denotes the superspace inverse vielbein, $\Omega_{A}{ }^{b c}$ the Lorentz connection, $\Phi_{A}$ the $\mathrm{U}(1)_{R}$ connection, $B_{A}$ the dilatation connection, and $\mathfrak{F}_{A B}$ the special superconformal connection.
The covariant derivatives (B1) obey the algebra

$$
\begin{gathered}
\left\{\nabla_{\alpha}, \nabla_{\beta}\right\}=0, \quad\left\{\nabla_{\alpha}, \bar{\nabla}_{\dot{\alpha}}\right\}=-2 \mathrm{i} \nabla_{\alpha \dot{\alpha}}, \\
{\left[\bar{\nabla}_{\dot{\alpha}}, \nabla_{\beta \dot{\beta}}\right]=-\mathrm{i} \varepsilon_{\dot{\alpha} \dot{\beta}}\left(2 W_{\beta}{ }^{\gamma \delta} M_{\gamma \delta}+\frac{1}{2} \nabla^{\alpha} W_{\alpha \beta \gamma} S^{\gamma}\right.} \\
\left.+\frac{1}{2} \nabla^{\alpha \dot{\gamma}} W_{\alpha \beta}{ }^{\gamma} K_{\gamma \dot{\gamma}}\right) .
\end{gathered}
$$

Here $W_{\alpha \beta \gamma}$ is the $\mathcal{N}=1$ super-Weyl tensor and is subject to the constraints:

$$
\begin{aligned}
K^{D} W_{\alpha \beta \gamma} & =0, \quad \bar{\nabla}^{\dot{\delta}} W_{\alpha \beta \gamma}=0, \\
\mathbb{D} W_{\alpha \beta \gamma} & =\frac{3}{2} W_{\alpha \beta \gamma}, \quad Y W_{\alpha \beta \gamma}=-W_{\alpha \beta \gamma},
\end{aligned}
$$

as well as the Bianchi identity

$$
B_{\alpha \dot{\alpha}}:=\mathrm{i} \nabla_{\dot{\alpha}}^{\beta} \nabla^{\gamma} W_{\alpha \beta \gamma}=\mathrm{i} \nabla_{\alpha}^{\dot{\beta}} \bar{\nabla}^{\dot{\gamma}} \bar{W}_{\dot{\alpha} \dot{\beta} \dot{\gamma}}=\bar{B}_{\alpha \dot{\alpha}},
$$

where the primary superfield $B_{\alpha \dot{\alpha}}$ is the super-Bach tensor and was introduced in [95], see also [35,43,87].

We remind the reader that a tensor superfield $\Psi$ (with suppressed indices) is said to be primary and of dimension $\Delta_{\Psi}$ and $\mathrm{U}(1)_{R}$ charge $q_{\Psi}$ if the following conditions hold:

$$
K^{B} \Psi=0, \quad \mathbb{D} \Psi=\Delta_{\Psi} \Psi, \quad Y \Psi=q_{\Psi} \Psi .
$$

Of particular importance are primary chiral superfields, which satisfy

$$
K^{B} \Psi=0, \quad \bar{\nabla} \dot{\beta} \Psi=0 .
$$

Requiring consistency of these constraints with the superconformal algebra yields highly nontrivial implications. Specifically, it must take the form $\Psi=\Psi_{\alpha(m)}$, and its $\mathrm{U}(1)_{R}$ charge and dimension are related as follows:

$$
q_{\Psi}=-\frac{2}{3} \Delta_{\Psi}
$$

The algebra (B2) is to be accompanied by the following (anti-)commutation relations: the $\mathrm{U}(1)_{R}$, dilatation and special conformal generators obey

$$
\begin{array}{lll}
{\left[Y, \nabla_{\alpha}\right]=\nabla_{\alpha},} & {\left[Y, \bar{\nabla}^{\dot{\alpha}}\right]=-\bar{\nabla}^{\dot{\alpha}},} & (\mathrm{B} 7 \mathrm{a}) \\
{\left[\mathbb{D}, \nabla_{\alpha \dot{\alpha}}\right]=\nabla_{\alpha \dot{\alpha}},} & {\left[\mathbb{D}, \nabla_{\alpha}\right]=\frac{1}{2} \nabla_{\alpha},} & {\left[\mathbb{D}, \bar{\nabla}^{\dot{\alpha}}\right]=\frac{1}{2} \bar{\nabla}^{\dot{\alpha}}}
\end{array}
$$

$\left[Y, S^{\alpha}\right]=-S^{\alpha}, \quad\left[Y, \bar{S}_{\dot{\alpha}}\right]=\bar{S}_{\dot{\alpha}}, \quad\left\{S_{\alpha}, \bar{S}_{\dot{\alpha}}\right\}=2 \mathrm{i} K_{\alpha \dot{\alpha}}$ 
$\left[\mathbb{D}, K_{\alpha \dot{\alpha}}\right]=-K_{\alpha \dot{\alpha}}, \quad\left[\mathbb{D}, S^{\alpha}\right]=-\frac{1}{2} S^{\alpha}, \quad\left[\mathbb{D}, \bar{S}_{\dot{\alpha}}\right]=-\frac{1}{2} \bar{S}_{\dot{\alpha}}$,

while the algebra of $K_{A}$ and $\nabla_{B}$ takes the form

$$
\begin{aligned}
& {\left[K_{\alpha \dot{\alpha}}, \nabla_{\beta \dot{\beta}}\right]=4\left(\varepsilon_{\dot{\alpha} \dot{\beta}} M_{\alpha \beta}+\varepsilon_{\alpha \beta} \bar{M}_{\dot{\alpha} \dot{\beta}}-\varepsilon_{\alpha \beta} \varepsilon_{\dot{\alpha} \dot{\beta}} \mathbb{D}\right),} \\
& \left\{S_{\alpha}, \nabla_{\beta}\right\}=\varepsilon_{\alpha \beta}(2 \mathbb{D}-3 Y)-4 M_{\alpha \beta}, \\
& \left\{\bar{S}_{\dot{\alpha}}, \bar{\nabla}_{\dot{\beta}}\right\}=-\varepsilon_{\dot{\alpha} \dot{\beta}}(2 \mathbb{D}+3 Y)+4 \bar{M}_{\dot{\alpha} \dot{\beta}}, \\
& {\left[K_{\alpha \dot{\alpha}}, \nabla_{\beta}\right]=-2 \mathrm{i} \varepsilon_{\alpha \beta} \bar{S}_{\dot{\alpha}}, \quad\left[K_{\alpha \dot{\alpha}}, \bar{\nabla}_{\dot{\beta}}\right]=2 \mathrm{i} \varepsilon_{\dot{\alpha} \dot{\beta}} S_{\alpha},} \\
& {\left[S_{\alpha}, \nabla_{\beta \dot{\beta}}\right]=2 \mathrm{i} \varepsilon_{\alpha \beta} \bar{\nabla}_{\dot{\beta}}, \quad\left[\bar{S}_{\dot{\alpha}}, \nabla_{\beta \dot{\beta}}\right]=-2 \mathrm{i} \varepsilon_{\dot{\alpha} \dot{\beta}} \nabla_{\beta},}
\end{aligned}
$$

where all other graded commutators vanish.

The chiral and full superspace integrals are related according to the rule

$$
\begin{aligned}
\int \mathrm{d}^{4} x \mathrm{~d}^{2} \theta \mathrm{d}^{2} \bar{\theta} E U & =-\frac{1}{4} \int \mathrm{d}^{4} x \mathrm{~d}^{2} \theta \mathcal{E} \bar{\nabla}^{2} U, \\
E^{-1} & =\operatorname{Ber}\left(E_{A}{ }^{M}\right),
\end{aligned}
$$

where $U$ is a primary real superfield of dimension +2 .

\section{APPENDIX C: $\mathcal{N}=2$ CONFORMAL SUPERSPACE}

This appendix reviews $\mathcal{N}=2$ conformal superspace, a formulation for off-shell $\mathcal{N}=2$ conformal supergravity developed by Butter [84] and then reformulated in [96].

We consider a curved $\mathcal{N}=2$ superspace $\mathcal{M}^{4 \mid 8}$ parametrized by local coordinates $z^{M}=\left(x^{m}, \theta_{l}^{\mu}, \bar{\theta}_{\dot{\mu}}^{l}\right)$. The structure group is chosen to be $\mathrm{SU}(2,2 \mid 2)$. The corresponding superalgebra is spanned by the Lorentz $M_{a b}$, translation $P_{A}=\left(P_{a}, Q_{\alpha}^{i}, \bar{Q}_{i}^{\dot{\alpha}}\right)$, dilatation $\mathbb{D}$, R-symmetry $Y$ and $J_{i j}$, and the special conformal $K^{A}=\left(K^{a}, S_{i}^{\alpha}, \bar{S}_{\dot{\alpha}}^{i}\right)$ generators. The covariant derivatives $\nabla_{A}=\left(\nabla_{a}, \nabla_{\alpha}^{i}, \bar{\nabla}_{i}^{\dot{\alpha}}\right)$ then have the form

$\nabla_{A}=E_{A}-\frac{1}{2} \Omega_{A}{ }^{a b} M_{a b}-\Phi_{A}{ }^{i j} J_{i j}-\mathrm{i} \Phi_{A} Y-B_{A} \mathbb{D}-\mathfrak{\mho}_{A B} K^{B}$.

As compared with (B1), we have introduced $\Phi_{A}{ }^{i j}$, the $\mathrm{SU}(2)_{R}$ connection. The corresponding generator acts on isospinors as follows:

$$
J^{i j} \chi^{k}=\varepsilon^{k(i} \chi^{j)} .
$$

The covariant derivatives $(\mathrm{C} 1)$ obey the algebra

$$
\begin{aligned}
\left\{\nabla_{\alpha}^{i}, \nabla_{\beta}^{j}\right\}= & 2 \varepsilon^{i j} \varepsilon_{\alpha \beta} \bar{W}_{\dot{\gamma} \dot{\delta}} \bar{M}^{\dot{\gamma} \dot{\delta}}+\frac{1}{2} \varepsilon^{i j} \varepsilon_{\alpha \beta} \bar{\nabla}_{\dot{\gamma} k} \bar{W}^{\dot{\gamma} \dot{\delta}} \bar{S}_{\dot{\delta}}^{k} \\
& -\frac{1}{2} \varepsilon^{i j} \varepsilon_{\alpha \beta} \nabla_{\dot{\gamma} \dot{\delta}} \bar{W}_{\dot{\gamma}}^{\dot{\delta}} K^{\gamma \dot{\gamma}}, \\
\left\{\nabla_{\alpha}^{i}, \bar{\nabla}_{j}^{\dot{\beta}}\right\}= & -2 \mathrm{i} \delta_{j}^{i} \nabla_{\alpha}^{\dot{\beta}}, \\
{\left[\nabla_{\alpha \dot{\alpha}}, \nabla_{\beta}^{i}\right]=} & -\mathrm{i} \varepsilon_{\alpha \beta} \bar{W}_{\dot{\alpha} \dot{\beta}} \bar{\nabla}^{\dot{\beta} i}-\frac{\mathrm{i}}{2} \varepsilon_{\alpha \beta} \bar{\nabla}^{\beta i} \bar{W}_{\dot{\alpha} \dot{\beta}} \mathbb{D} \\
& -\frac{\mathrm{i}}{4} \varepsilon_{\alpha \beta} \bar{\nabla}^{\dot{\beta} i} \bar{W}_{\dot{\alpha} \dot{\beta}} Y+\mathrm{i} \varepsilon_{\alpha \beta} \bar{\nabla}_{j}^{\dot{\beta}} \bar{W}_{\dot{\alpha} \dot{\beta}} J^{i j} \\
& -\mathrm{i} \varepsilon_{\alpha \beta} \bar{\nabla}_{\dot{\beta}}^{i} \bar{W}_{\dot{\gamma} \dot{\alpha}} \bar{M}^{\dot{\beta} \dot{\gamma}}-\frac{\mathrm{i}}{4} \varepsilon_{\alpha \beta} \bar{\nabla}_{\dot{\alpha}}^{i} \bar{\nabla}_{k}^{\dot{\beta}} \bar{W}_{\dot{\beta} \dot{\gamma}} \bar{S}^{\dot{\gamma} k} \\
& +\frac{1}{2} \varepsilon_{\alpha \beta} \nabla^{\gamma \dot{\beta}} \bar{W}_{\dot{\alpha} \dot{\beta}} S_{\gamma}^{i}+\frac{\mathrm{i}}{4} \varepsilon_{\alpha \beta} \bar{\nabla}_{\dot{\alpha}}^{i} \nabla_{\dot{\gamma}}^{\gamma} \bar{W}^{\dot{\gamma} \dot{\beta}} K_{\gamma \dot{\beta}} .
\end{aligned}
$$

Here $W_{\alpha \beta}$ is the $\mathcal{N}=2$ super-Weyl tensor and is subject to the constraints:

$$
\begin{aligned}
K^{C} W_{\alpha \beta} & =0, \quad \bar{\nabla}_{k}^{\dot{\gamma}} W_{\alpha \beta}=0, \quad \mathbb{D} W_{\alpha \beta}=W_{\alpha \beta}, \\
Y W_{\alpha \beta} & =-W_{\alpha \beta} .
\end{aligned}
$$

We also find that $W_{\alpha \beta}$ obeys the Bianchi identity

$$
B=\nabla_{\alpha \beta} W^{\alpha \beta}=\bar{\nabla}^{\dot{\alpha} \dot{\beta}} \bar{W}_{\dot{\alpha} \dot{\beta}}=\bar{B},
$$

where the primary superfield $B$ is the $\mathcal{N}=2$ super-Bach tensor. We remind the reader that a superfield $\Phi$ (with suppressed indices) is said to be primary of dimension $\Delta_{\Psi}$ and $\mathrm{U}(1)_{R}$ charge $q_{\Psi}$ if the following conditions hold:

$$
K^{B} \Psi=0, \quad \mathbb{D} \Psi=\Delta_{\Psi} \Psi, \quad Y \Psi=q_{\Psi} \Psi .
$$

Of particular importance are primary chiral superfields, which satisfy

$$
K^{B} \Psi=0, \quad \bar{\nabla}_{j}^{\dot{\beta}} \Psi=0 .
$$

The consistency of these constraints with the superconformal algebra leads to highly nontrivial implications. In particular, it can carry no isospinor or dotted spinor indices, $\Psi=\Psi_{\alpha(m)}$, and its $\mathrm{U}(1)_{R}$ charge and dimension are related as follows:

$$
q_{\Psi}=-2 \Delta_{\Psi}
$$

Further, we note that for any primary tensor superfield $\mathfrak{U}_{\alpha(m)}$ with the property $q_{\mathfrak{U}}=-2 \Delta_{\mathfrak{U}}$, the following object 


$$
\Psi_{\alpha(m)}=\bar{\nabla}^{4} \mathfrak{U}_{\alpha(m)} \equiv \frac{1}{48} \bar{\nabla}^{i j} \bar{\nabla}_{i j} \mathfrak{U}_{\alpha(m)}
$$

is both primary and chiral in conformally flat backgrounds [84,97].

The chiral and full superspace integrals are related according to the rule

$$
\int \mathrm{d}^{4} x \mathrm{~d}^{4} \theta \mathrm{d}^{4} \bar{\theta} \boldsymbol{E} U=\int \mathrm{d}^{4} x \mathrm{~d}^{4} \theta \mathcal{E} \bar{\nabla}^{4} U, \quad \boldsymbol{E}^{-1}=\operatorname{Ber}\left(E_{A}{ }^{M}\right),
$$

where $U$ is a primary real dimension-0 superfield.

For further details regarding $\mathcal{N}=2$ conformal superspace, we refer the reader to the original work [84], as well as [52,96].

\section{APPENDIX D: ELIMINATION OF AUXILIARY VARIABLES}

This appendix is devoted to a derivation of the conformal $\mathrm{U}(1)$ duality-invariant CHS models (2.37) via the auxiliary variable formalism introduced in Sec. II C. We emphasize that the latter is a higher-spin generalization of the IvanovZupnik approach [6-8].

Consider the following action functional:

$$
\begin{aligned}
S^{(s)}[\mathcal{C}, \overline{\mathcal{C}}, \rho, \bar{\rho}]= & (-1)^{s} \int \mathrm{d}^{4} x e\left\{2 \rho \mathcal{C}-\rho^{2}-\frac{1}{2} \mathcal{C}^{2}\right\} \\
& + \text { c.c. }+\beta \int \mathrm{d}^{4} x e \sqrt{\rho^{2} \bar{\rho}^{2}},
\end{aligned}
$$

where one should keep in mind the definitions of Sec. II. It is clear that (D1) is both conformal and U(1) duality invariant. Varying this action with respect to the auxiliary variable $\rho^{\alpha(2 s)}$ yields

$$
\rho_{\alpha(2 s)}=\mathcal{C}_{\alpha(2 s)}+\frac{(-1)^{s} \beta}{2} \frac{\rho_{\alpha(2 s)} \bar{\rho}^{2}}{\sqrt{\rho^{2} \bar{\rho}^{2}}}
$$

Employing this result, it is possible to integrate out $\rho_{\alpha(2 s)}$. As a result, we obtain the self-dual model

$$
\begin{aligned}
S^{(s)}[\mathcal{C}, \overline{\mathcal{C}}]= & \frac{(-1)^{s}}{2} \frac{1+(\beta / 2)^{2}}{1-(\beta / 2)^{2}} \int \mathrm{d}^{4} x e\left\{\mathcal{C}^{2}+\overline{\mathcal{C}}^{2}\right\} \\
& +\frac{\beta}{1-(\beta / 2)^{2}} \int \mathrm{d}^{4} x e \sqrt{\mathcal{C}^{2} \overline{\mathcal{C}}^{2}}
\end{aligned}
$$

Now, upon making the identification

$$
\cosh \gamma=\frac{1+(\beta / 2)^{2}}{1-(\beta / 2)^{2}} \Leftrightarrow \sinh \gamma=\frac{\beta}{1-(\beta / 2)^{2}},
$$

it is clear that (D3) coincides with (2.37), which concludes our analysis. It is important to note that, in the $s=1$ case, this computation was first performed in [28].

\section{APPENDIX E: OVERALL SIGNS FOR FREE (S)CHS ACTIONS}

In this appendix we show that the overall signs of the free CHS actions [32-34]

$$
S^{(s)}[\mathcal{C}, \overline{\mathcal{C}}]=\frac{(-1)^{s}}{2} \int \mathrm{d}^{4} x e\left\{\mathcal{C}^{\alpha(2 s)} \mathcal{C}_{\alpha(2 s)}+\text { c.c. }\right\}
$$

can be fixed by making use of supersymmetry considerations in conjunction with the known action of Maxwell theory for $s=1$,

$$
\begin{aligned}
S^{(1)}[\mathcal{C}, \overline{\mathcal{C}}] & =-\frac{1}{2} \int \mathrm{d}^{4} x e\left\{\mathcal{C}^{\alpha(2)} \mathcal{C}_{\alpha(2)}+\text { c.c. }\right\} \\
& =-\frac{1}{4} \int \mathrm{d}^{4} x e C^{a b} C_{a b},
\end{aligned}
$$

where $C_{a b}=\nabla_{a} h_{b}-\nabla_{b} h_{a}$. Moreover, similar arguments allow us to correctly fix the overall signs of the free $\mathcal{N}=1$ and $\mathcal{N}=2$ SCHS actions.

The overall sign in (E1) is also fixed by identifying the action $S^{(s)}[\mathcal{C}, \overline{\mathcal{C}}]$ with the induced one obtained by computing the logarithmically divergent part of the effective action of a conformal scalar field coupled to background conformal higher-spin fields [98].

\section{1. $\mathcal{N}=1$ actions}

Consider the chiral field strength $\mathcal{W}_{\alpha(2 s+1)}$ and introduce its bosonic components

$$
\begin{aligned}
\mathcal{C}_{\alpha(2 s)} & :=\left.\frac{1}{4} \sqrt{\frac{2 s+1}{s+1}} \nabla^{\beta} \mathcal{W}_{\beta \alpha(2 s)}\right|_{\theta=0}, \\
\mathcal{C}_{\alpha(2 s+2)} & :=\left.\frac{\sqrt{2} \mathrm{i}}{4} \nabla_{\left(\alpha_{1}\right.} \mathcal{W}_{\left.\alpha_{2} \ldots \alpha_{2 s+2}\right)}\right|_{\theta=0},
\end{aligned}
$$

which have been defined such that Bianchi identity (2.6) holds both for field strengths. We then compute the bosonic part of the SCHS action $[35,43]$

$$
\begin{aligned}
& S^{(s)}[\mathcal{W}, \overline{\mathcal{W}}] \\
& :=\frac{z_{s}}{4} \int \mathrm{d}^{4} x \mathrm{~d}^{2} \theta \mathcal{E} \mathcal{W}^{\alpha(2 s+1)} \mathcal{W}_{\alpha(2 s+1)}+\text { c.c. } \\
& =-\left.\frac{z_{s}}{16} \int \mathrm{d}^{4} x e \nabla^{2}\left(\mathcal{W}^{\alpha(2 s+1)} \mathcal{W}_{\alpha(2 s+1)}\right)\right|_{\theta=\bar{\theta}=0}+\text { c.c. } \\
& =z_{s} \int \mathrm{d}^{4} x e\left(\mathcal{C}^{\alpha(2 s)} \mathcal{C}_{\alpha(2 s)}-\mathcal{C}^{\alpha(2 s+2)} \mathcal{C}_{\alpha(2 s+2)}\right)+\cdots+\text { c.c. }
\end{aligned}
$$


where the ellipses denote the fermonic sector, which is irrelevant to our analysis. For the overall signs of the component actions present in (E4) to agree with those of (E1), we require $z_{s}=(-1)^{s}$. Therefore, the $\mathcal{N}=1$ SCHS actions take the form $[35,43]$

$$
S^{(s)}[\mathcal{W}, \overline{\mathcal{W}}]=\frac{(-1)^{s}}{4} \int \mathrm{d}^{4} x \mathrm{~d}^{2} \theta \mathcal{E} \mathcal{W}^{\alpha(2 s+1)} \mathcal{W}_{\alpha(2 s+1)}+\text { c.c. }
$$

\section{2. $\mathcal{N}=2$ actions}

Consider the chiral field strength $\mathcal{W}_{\alpha(2 s+2)}$. It contains two $\mathcal{N}=1$ fermionic superfields in its multiplet

$$
\begin{aligned}
\mathcal{W}_{\alpha(2 s+1)} & :=\left.\frac{\mathrm{i}}{2} \sqrt{\frac{s+1}{2 s+3}} \nabla^{\beta 2} \mathcal{W}_{\alpha(2 s+1) \beta}\right|_{\theta_{\underline{2}}=0}, \\
\mathcal{W}_{\alpha(2 s+3)}: & :=\left.\frac{\sqrt{2}}{4} \nabla_{\left(\alpha_{1}\right.}^{2} \mathcal{W}_{\left.\alpha_{2} \ldots \alpha_{2 s+3}\right)}\right|_{\theta_{\underline{2}}=0},
\end{aligned}
$$

which have been defined such that the Bianchi identity (3.7) holds for both field strengths. Next, we reduce the $\mathcal{N}=2$ SCHS action [52] to $\mathcal{N}=1$ superspace

$$
\begin{aligned}
S^{(s)}[\mathcal{W}, \overline{\mathcal{W}}] \\
:=\frac{z_{s}}{4} \int \mathrm{d}^{4} x \mathrm{~d}^{4} \theta \mathcal{E} \mathcal{W}^{\alpha(2 s+1)} \mathcal{W}_{\alpha(2 s+1)}+\text { c.c. } \\
=-\frac{z_{s}}{16} \int \mathrm{d}^{4} x \mathrm{~d}^{2} \theta \mathcal{E} \nabla^{\alpha 2}-\left.\nabla_{\bar{\alpha}}^{2}\left(\mathcal{W}^{\alpha(2 s+1)} \mathcal{W}_{\alpha(2 s+1)}\right)\right|_{\theta^{2}=\bar{\theta}_{\underline{2}}=0}+\text { c.c. } \\
=z_{s} \int \mathrm{d}^{4} x \mathrm{~d}^{2} \theta \mathcal{E}\left(\mathcal{W}^{\alpha(2 s+1)} \mathcal{W}_{\alpha(2 s+1)}-\mathcal{W}^{\alpha(2 s+3)} \mathcal{W}_{\alpha(2 s+3)}\right) \\
\quad+\cdots+\text { c.c. }
\end{aligned}
$$

where the ellipses denotes the sector containing integer superspin field strengths, which is irrelevant to our analysis. For the overall signs of the $\mathcal{N}=1$ actions present in (E7) to agree with those of (E5), we require $z_{s}=(-1)^{s}$. Therefore, the $\mathcal{N}=2$ SCHS actions take the form [52]

$S^{(s)}[\mathcal{W}, \overline{\mathcal{W}}]=\frac{(-1)^{s}}{4} \int \mathrm{d}^{4} x \mathrm{~d}^{4} \theta \mathcal{E} \mathcal{W}^{\alpha(2 s+2)} \mathcal{W}_{\alpha(2 s+2)}+$ c.c.
[1] M. K. Gaillard and B. Zumino, Duality rotations for interacting fields, Nucl. Phys. B193, 221 (1981).

[2] G. W. Gibbons and D. A. Rasheed, Electric-magnetic duality rotations in nonlinear electrodynamics, Nucl. Phys. B454, 185 (1995).

[3] G. W. Gibbons and D. A. Rasheed, SL(2,R) invariance of non-linear electrodynamics coupled to an axion and a dilaton, Phys. Lett. B 365, 46 (1996).

[4] M. K. Gaillard and B. Zumino, Self-duality in nonlinear electromagnetism, in Supersymmetry and Quantum Field Theory, edited by J. Wess and V. P. Akulov (Springer Verlag, Berlin, 1998), pp. 121-129.

[5] M. K. Gaillard and B. Zumino, Nonlinear electromagnetic self-duality and Legendre transformations, in Duality and Supersymmetric Theories, edited by D. I. Olive and P. C. West (Cambridge University Press, Cambridge, England, 1999), pp. 33-48.

[6] E. A. Ivanov and B. M. Zupnik, $N=3$ supersymmetric Born-Infeld theory, Nucl. Phys. B618, 3 (2001).

[7] E. A. Ivanov and B. M. Zupnik, New representation for Lagrangians of self-dual nonlinear electrodynamics, in Supersymmetries and Quantum Symmetries. Proceedings of the 16th Max Born Symposium, SQS'01: Karpacz, Poland, September 21-25, 2001, edited by E. Ivanov (Joint Institute for Nuclear Research, Dubna, 2002), pp. 235-250.

[8] E. A. Ivanov and B. M. Zupnik, New approach to nonlinear electrodynamics: Dualities as symmetries of interaction, Yad. Fiz. 67, 2212 (2004) [Phys. At. Nucl. 67, 2188 (2004)].
[9] S. M. Kuzenko and S. Theisen, Supersymmetric duality rotations, J. High Energy Phys. 03 (2000) 034.

[10] S. M. Kuzenko and S. Theisen, Nonlinear self-duality and supersymmetry, Fortschr. Phys. 49, 273 (2001).

[11] S. M. Kuzenko and S. A. McCarthy, Nonlinear selfduality and supergravity, J. High Energy Phys. 02 (2003) 038.

[12] S. M. Kuzenko and S. A. McCarthy, On the component structure of $N=1$ supersymmetric nonlinear electrodynamics, J. High Energy Phys. 05 (2005) 012.

[13] S. M. Kuzenko, Nonlinear self-duality in $N=2$ supergravity, J. High Energy Phys. 06 (2012) 012.

[14] S. V. Ketov, A manifestly $N=2$ supersymmetric BornInfeld action, Mod. Phys. Lett. A 14, 501 (1999); BornInfeld-Goldstone superfield actions for gauge-fixed D5- and D3-branes in 6d, Nucl. Phys. B553, 250 (1999).

[15] S. Bellucci, E. Ivanov, and S. Krivonos, $N=2$ and $N=4$ supersymmetric Born-Infeld theories from nonlinear realizations, Phys. Lett. B 502, 279 (2001).

[16] S. Bellucci, E. Ivanov, and S. Krivonos, Towards the complete $N=2$ superfield Born-Infeld action with partially broken $N=4$ supersymmetry, Phys. Rev. D 64, 025014 (2001).

[17] J. Broedel, J. J. M. Carrasco, S. Ferrara, R. Kallosh, and R. Roiban, $N=2$ supersymmetry and U(1)-duality, Phys. Rev. D 85, 125036 (2012).

[18] J. J. M. Carrasco and R. Kallosh, Hidden supersymmetry may imply duality invariance, arXiv:1303.5663. 
[19] E. A. Ivanov and B. M. Zupnik, Self-dual $\mathcal{N}=2$ BornInfeld theory through auxiliary superfields, J. High Energy Phys. 05 (2014) 061.

[20] S. Cecotti and S. Ferrara, Supersymmetric Born-Infeld Lagrangians, Phys. Lett. B 187, 335 (1987).

[21] D. Brace, B. Morariu, and B. Zumino, Duality invariant Born-Infeld theory, in The Many Faces of the Superworld: Yury Golfand Memorial Volume, edited by M. Shifman (World Scientific, Singapore, 2000), pp. 103-110.

[22] J. Bagger and A. Galperin, A new Goldstone multiplet for partially broken supersymmetry, Phys. Rev. D 55, 1091 (1997).

[23] M. Roček and A. A. Tseytlin, Partial breaking of global $\mathrm{D}=4$ supersymmetry, constrained superfields, and 3-brane actions, Phys. Rev. D 59, 106001 (1999).

[24] S. M. Kuzenko and G. Tartaglino-Mazzucchelli, Nilpotent chiral superfield in $N=2$ supergravity and partial rigid supersymmetry breaking, J. High Energy Phys. 03 (2016) 092.

[25] G. Festuccia and N. Seiberg, Rigid supersymmetric theories in curved superspace, J. High Energy Phys. 06 (2011) 114.

[26] S. M. Kuzenko, Duality rotations in supersymmetric nonlinear electrodynamics revisited, J. High Energy Phys. 03 (2013) 153.

[27] E. Ivanov, O. Lechtenfeld, and B. Zupnik, Auxiliary superfields in $N=1$ supersymmetric self-dual electrodynamics, J. High Energy Phys. 05 (2013) 133.

[28] S. M. Kuzenko, Superconformal duality-invariant models and $\mathcal{N}=4$ SYM effective action, arXiv:2106.07173.

[29] I. Bandos, K. Lechner, D. Sorokin, and P. K. Townsend, A nonlinear duality-invariant conformal extension of Maxwell's equations, Phys. Rev. D 102, 121703 (2020).

[30] B. P. Kosyakov, Nonlinear electrodynamics with the maximum allowable symmetries, Phys. Lett. B 810, 135840 (2020).

[31] I. Bandos, K. Lechner, D. Sorokin, and P. K. Townsend, ModMax meets Susy, arXiv:2106.07547.

[32] E. S. Fradkin and A. A. Tseytlin, Conformal supergravity, Phys. Rep. 119, 233 (1985).

[33] E.S. Fradkin and V. Y. Linetsky, Cubic interaction in conformal theory of integer higher-spin fields in four dimensional space-time, Phys. Lett. B 231, 97 (1989).

[34] E. S. Fradkin and V. Y. Linetsky, Superconformal higher spin theory in the cubic approximation, Nucl. Phys. B350, 274 (1991).

[35] S. M. Kuzenko and M. Ponds, Conformal geometry and (super)conformal higher-spin gauge theories, J. High Energy Phys. 05 (2019) 113.

[36] I. Bialynicki-Birula, Nonlinear electrodynamics: Variations on a theme by Born and Infeld, in Quantum Theory of Particles and Fields, edited by B. Jancewicz and J. Lukierski (World Scientific, 1983), pp. 31-48.

[37] S. Deser, Scale invariance and gravitational coupling, Ann. Phys. (N.Y.) 59, 248 (1970).

[38] B. Zumino, Effective Lagrangians and broken symmetries, in Lectures on Elementary Particles and Quantum Field Theory, Vol. 2, edited by S. Deser, M. Grisaru, and H. Pendleton (MIT Press, Cambridge, MA, 1970), pp. 437-500.
[39] M. Kaku and P. K. Townsend, Poincaré supergravity as broken superconformal gravity, Phys. Lett. 76B, 54 (1978).

[40] M. Born and L. Infeld, Foundations of the new field theory, Proc. R. Soc. A 144, 425 (1934).

[41] I. Bandos, K. Lechner, D. Sorokin, and P. K. Townsend, On p-form gauge theories and their conformal limits, J. High Energy Phys. 03 (2021) 022.

[42] R. Penrose and W. Rindler, Spinors and Space-Time: Volume 2, Spinor and Twistor Methods in Space-Time Geometry (Cambridge University Press, Cambridge, England, 1986).

[43] S. M. Kuzenko, R. Manvelyan, and S. Theisen, Off-shell superconformal higher spin multiplets in four dimensions, J. High Energy Phys. 07 (2017) 034.

[44] V. Ogievetsky and E. Sokatchev, On vector superfield generated by supercurrent, Nucl. Phys. B124, 309 (1977).

[45] S. Ferrara and B. Zumino, Structure of linearized supergravity and conformal supergravity, Nucl. Phys. B134, 301 (1978).

[46] S. Ferrara and B. Zumino, Supergauge invariant Yang-Mills theories, Nucl. Phys. B79, 413 (1974).

[47] J. Wess and B. Zumino, Superfield Lagrangian for supergravity, Phys. Lett. 74B, 51 (1978).

[48] S. M. Kuzenko, M. Ponds, and E. S. N. Raptakis, New locally (super)conformal gauge models in Bach-flat backgrounds, J. High Energy Phys. 08 (2020) 068.

[49] S. Ferrara, L. Girardello, T. Kugo, and A. Van Proeyen, Relation between different auxiliary field formulations of $N=1$ supergravity coupled to matter, Nucl. Phys. B223, 191 (1983).

[50] V. Rittenberg and E. Sokatchev, Decomposition of extended superfields into irreducible representations of supersymmetry, Nucl. Phys. B193, 477 (1981).

[51] S. J. Gates Jr., M. T. Grisaru, M. Roček, and W. Siegel, Superspace, or one thousand and one lessons in supersymmetry, Front. Phys. 58, 1 (1983).

[52] S. M. Kuzenko and E. S. N. Raptakis, $\mathcal{N}=2$ superconformal higher-spin gauge theories in four dimensions, arXiv: 2104.10416.

[53] R. Grimm, M. Sohnius, and J. Wess, Extended supersymmetry and gauge theories, Nucl. Phys. B133, 275 (1978).

[54] L. Mezincescu, On the superfield formulation of $\mathrm{O}(2)$ supersymmetry, Dubna preprint, Report No. JINR-P2-12572, 1979.

[55] P. S. Howe, K. S. Stelle, and P. K. Townsend, Supercurrents, Nucl. Phys. B192, 332 (1981).

[56] D. Butter and S. M. Kuzenko, New higher-derivative couplings in $4 \mathrm{D} N=2$ supergravity, J. High Energy Phys. 03 (2011) 047.

[57] D. Butter and S. M. Kuzenko, $N=2$ AdS supergravity and supercurrents, J. High Energy Phys. 07 (2011) 081.

[58] S. M. Kuzenko and S. Theisen, Correlation functions of conserved currents in $N=2$ superconformal theory, Classical Quantum Gravity 17, 665 (2000).

[59] I. L. Buchbinder, S. M. Kuzenko, and A. A. Tseytlin, On low-energy effective actions in $N=2,4$ superconformal theories in four dimensions, Phys. Rev. D 62, 045001 (2000). 
[60] S. Deser and C. Teitelboim, Duality transformations of Abelian and non-Abelian gauge fields, Phys. Rev. D 13, 1592 (1976).

[61] S. Deser, Off-shell electromagnetic duality invariance, J. Phys. A 15, 1053 (1982).

[62] M. Henneaux and C. Teitelboim, Duality in linearized gravity, Phys. Rev. D 71, 024018 (2005).

[63] S. Deser and D. Seminara, Duality invariance of all free bosonic and fermionic gauge fields, Phys. Lett. B 607, 317 (2005).

[64] C. Bunster and M. Henneaux, The action for twisted selfduality, Phys. Rev. D 83, 125015 (2011).

[65] C. Bunster and M. Henneaux, Supersymmetric electricmagnetic duality as a manifest symmetry of the action for super-Maxwell theory and linearized supergravity, Phys. Rev. D 86, 065018 (2012).

[66] C. Bunster, M. Henneaux, and S. Hörtner, Twisted selfduality for linearized gravity in D dimensions, Phys. Rev. D 88, 064032 (2013).

[67] V. I. Ogievetsky and I. V. Polubarinov, The notoph and its possible interactions, Sov. J. Nucl. Phys. 4 (1967) 156; reprinted in Supersymmetries and Quantum Symmetries, edited by J. Wess and E. A. Ivanov (Springer, New York, 1999), pp. 391-396.

[68] M. Kalb and P. Ramond, Classical direct interstring action, Phys. Rev. D 9, 2273 (1974).

[69] E. Cremmer and J. Scherk, Spontaneous dynamical breaking of gauge symmetry in dual models, Nucl. Phys. B72, 117 (1974).

[70] T. Curtright and P. G. O. Freund, Massive dual fields, Nucl. Phys. B172, 413 (1980).

[71] D. Z. Freedman and P. K. Townsend, Antisymmetric tensor gauge theories and nonlinear sigma models, Nucl. Phys. B177, 282 (1981).

[72] T. Curtright, Generalized gauge fields, Phys. Lett. 165B, 304 (1985).

[73] E. Fradkin and A. A. Tseytlin, Quantum equivalence of dual field theories, Ann. Phys. (N.Y.) 162, 31 (1985).

[74] C. M. Hull, Duality in gravity and higher spin gauge fields, J. High Energy Phys. 09 (2001) 027.

[75] X. Bekaert and N. Boulanger, Tensor gauge fields in arbitrary representations of GL(D,R): Duality and Poincare lemma, Commun. Math. Phys. 245, 27 (2004).

[76] N. Boulanger, S. Cnockaert, and M. Henneaux, A note on spin s duality, J. High Energy Phys. 06 (2003) 060.

[77] A. S. Matveev and M. A. Vasiliev, On dual formulation for higher spin gauge fields in (A)dS(d), Phys. Lett. B 609, 157 (2005).

[78] Y. M. Zinoviev, On dual formulations of massive tensor fields, J. High Energy Phys. 10 (2005) 075.

[79] K. Hinterbichler and A. Joyce, Manifest duality for partially massless higher spins, J. High Energy Phys. 09 (2016) 141.

[80] N. Boulanger, A. Campoleoni, and I. Cortese, Dual actions for massless, partially-massless and massive gravitons in (A)dS, Phys. Lett. B 782, 285 (2018).
[81] P. Aschieri, S. Ferrara, and B. Zumino, Duality rotations in nonlinear electrodynamics and in extended supergravity, Riv. Nuovo Cimento 31, 625 (2008).

[82] E. A. Ivanov, O. Lechtenfeld, and B. M. Zupnik, Auxiliary tensor fields for $\operatorname{Sp}(2, \mathbb{R})$ self-duality, J. High Energy Phys. 03 (2015) 123.

[83] D. Butter, $N=1$ conformal superspace in four dimensions, Ann. Phys. (Amsterdam) 325, 1026 (2010).

[84] D. Butter, $N=2$ conformal superspace in four dimensions, J. High Energy Phys. 10 (2011) 030.

[85] I. A. Bandos, E. Ivanov, J. Lukierski, and D. Sorokin, On the superconformal flatness of AdS superspaces, J. High Energy Phys. 06 (2002) 040.

[86] S. M. Kuzenko and G. Tartaglino-Mazzucchelli, Field theory in 4D $N=2$ conformally flat superspace, J. High Energy Phys. 10 (2008) 001.

[87] I. L. Buchbinder and S. M. Kuzenko, Ideas and Methods of Supersymmetry and Supergravity or a Walk Through Superspace (IOP, Bristol, 1995) (Revised Edition: 1998).

[88] S. M. Kuzenko and A. G. Sibiryakov, Free massless higher-superspin superfields on the anti-de Sitter superspace, Yad. Fiz. 57, 1326 (1994) [Phys. At. Nucl. 57, 1257 (1994)].

[89] M. A. Vasiliev, Bosonic conformal higher-spin fields of any symmetry, Nucl. Phys. B829, 176 (2010).

[90] S. M. Kuzenko and E. S. N. Raptakis, Symmetries of supergravity backgrounds and supersymmetric field theory, J. High Energy Phys. 04 (2020) 133.

[91] S. M. Kuzenko, M. Ponds, and E. S. N. Raptakis, Generalized superconformal higher-spin multiplets, J. High Energy Phys. 03 (2021) 183.

[92] M. Kaku, P. K. Townsend, and P. van Nieuwenhuizen, Gauge theory of the conformal and superconformal group, Phys. Lett. 69B, 304 (1977).

[93] D. Butter, S. M. Kuzenko, J. Novak, and G. TartaglinoMazzucchelli, Conformal supergravity in three dimensions: New off-shell formulation, J. High Energy Phys. 09 (2013) 072.

[94] J. Wess and J. Bagger, Supersymmetry and Supergravity (Princeton University Press, Princeton, 1983) (Second Edition 1992).

[95] I. L. Buchbinder and S. M. Kuzenko, Quantization of the classically equivalent theories in the superspace of simple supergravity and quantum equivalence, Nucl. Phys. B308, 162 (1988).

[96] D. Butter and J. Novak, Component reduction in $N=2$ supergravity: The vector, tensor, and vector-tensor multiplets, J. High Energy Phys. 05 (2012) 115.

[97] S. M. Kuzenko and G. Tartaglino-Mazzucchelli, Different representations for the action principle in 4D $N=2$ supergravity, J. High Energy Phys. 04 (2009) 007.

[98] M. Beccaria and A. A. Tseytlin, On induced action for conformal higher spins in curved background, Nucl. Phys. B919, 359 (2017). 